\title{
Pulse Duration of Seeded Free-Electron Lasers
}

Paola Finetti, ${ }^{1}$ Hauke Höppner, ${ }^{2,3,4}$ Enrico Allaria, ${ }^{1}$ Carlo Callegari, ${ }^{1}$ Flavio Capotondi, ${ }^{1}$ Paolo Cinquegrana, ${ }^{1}$ Marcello Coreno, ${ }^{5,1}$ Riccardo Cucini, ${ }^{1}$ Miltcho B. Danailov, ${ }^{1}$ Alexander Demidovich, ${ }^{1}$ Giovanni De Ninno, ${ }^{1,6}$ Michele Di Fraia, ${ }^{7,1}$ Raimund Feifel, ${ }^{8}$ Eugenio Ferrari, ${ }^{1}$ Lars Fröhlich, ${ }^{1,2}$ David Gauthier, ${ }^{1}$ Torsten Golz, ${ }^{2}$ Cesare Grazioli, ${ }^{9,5}$ Yun Kai, ${ }^{3,4}$ Gabor Kurdi, ${ }^{1}$ Nicola Mahne, ${ }^{1}$ Michele Manfredda, ${ }^{1}$ Nikita Medvedev, ${ }^{10,11,12}$ Ivaylo P. Nikolov, ${ }^{1}$ Emanuele Pedersoli, ${ }^{1}$ Giuseppe Penco, ${ }^{1}$ Oksana Plekan, ${ }^{1}$ Mark J. Prandolini,,${ }^{13,14}$ Kevin C. Prince, ${ }^{1,15,16}$ Lorenzo Raimondi, ${ }^{1}$ Primoz Rebernik, ${ }^{1}$ Robert Riedel ${ }_{1}^{13,14}$ Eleonore Roussel,${ }^{1}$ Paolo Sigalotti, ${ }^{1}$ Richard Squibb, ${ }^{8}$ Nikola Stojanovic, ${ }^{2}$ Stefano Stranges, ${ }^{17,16}$ Cristian Svetina, ${ }^{1,18}$ Takanori Tanikawa, ${ }^{2}$ Ulrich Teubner, ${ }^{3,4}$ Victor Tkachenko, ${ }^{10}$ Sven Toleikis, ${ }^{2}$ Marco Zangrando, ${ }^{16,1}$ Beata Ziaja, ${ }^{10,1}$ Franz Tavella, ${ }^{20}$ and Luca Giannessi ${ }^{21,1}$

${ }^{1}$ Elettra-Sincrotrone Trieste S.C.p.A, 34149 Basovizza, Trieste, Italy

${ }^{2}$ Deutsches Elektronen-Synchrotron, Notkestraße 85, 22607 Hamburg, Germany

${ }^{3}$ Institut für Physik, Carl von Ossietzky Universität, 26111 Oldenburg, Germany

${ }^{4}$ Institut für Laser und Optik, Hochschule Emden/Leer-University of Applied Sciences, Constantiaplatz 4, 26723 Emden, Germany

${ }^{5}$ CNR-ISM, 34139 Basovizza, Trieste Italy

${ }^{6}$ Laboratory of Quantum Optics, University of Nova Gorica, Nova Gorica 5001, Slovenia

${ }^{7}$ Department of Physics, University of Trieste, 34127 Trieste, Italy

${ }^{8}$ University of Gothenburg, Department of Physics, Origovägen 6B,412 96 Gothenburg, Sweden

${ }^{9}$ Department of Chemical and Pharmaceutical Sciences, University of Trieste, 34127 Trieste, Italy

${ }^{10}$ Center for Free-Electron Laser Science/Deutsches Elektronen-Synchrotron DESY, Notkestraße 85, 22607 Hamburg, Germany

${ }^{11}$ Department of Radiation and Chemical Physics, Institute of Physics, Czech Academy of Sciences, Na Slovance 2, 18221 Prague 8, Czech Republic

${ }^{12}$ Laser Plasma Department, Institute of Plasma Physics, Czech Academy of Sciences, Za Slovankou 3 , 18200 Prague 8, Czech Republic

${ }^{13}$ Helmholtz-Institut Jena, Fröbelstieg 3, 07743 Jena, Germany

${ }^{14}$ Class 5 Photonics GmbH, Notkestraße 85, 22607 Hamburg, Germany

${ }^{15}$ Molecular Model Discovery Laboratory, Department of Chemistry and Biotechnology, Swinburne University of Technology, Melbourne, 3122, Australia

${ }^{16}$ CNR-IOM, Laboratorio Nazionale TASC, 34149 Basovizza, Trieste, Italy

${ }^{17}$ Dipartimento di Chimica e Tecnologie del Farmaco, Università la Sapienza, 00185 Roma, Italy

${ }^{18}$ Graduate School of Nanotechnology, University of Trieste, 34127 Trieste, Italy

${ }^{19}$ Institute of Nuclear Physics, Polish Academy of Sciences, Radzikowskiego 152, 31-142 Krakow, Poland

${ }^{20}$ SLAC National Accelerator Laboratory, 2575 Sand Hill Road, California 94025, USA

${ }^{21}$ ENEA, via Enrico Fermi 45, 00044 Frascati Roma, Italy

(Received 21 December 2016; published 16 June 2017)

The pulse duration, and, more generally, the temporal intensity profile of free-electron laser (FEL) pulses, is of utmost importance for exploring the new perspectives offered by FELs; it is a nontrivial experimental parameter that needs to be characterized. We measured the pulse shape of an extreme ultraviolet externally seeded FEL operating in high-gain harmonic generation mode. Two different methods based on the cross-correlation of the FEL pulses with an external optical laser were used. The two methods, one capable of single-shot performance, may both be implemented as online diagnostics in FEL facilities. The measurements were carried out at the seeded FEL facility FERMI. The FEL temporal pulse characteristics were measured and studied in a range of FEL wavelengths and machine settings, and they were compared to the predictions of a theoretical model. The measurements allowed a direct observation of the pulse lengthening and splitting at saturation, in agreement with the proposed theory.

DOI: 10.1103/PhysRevX.7.021043

Published by the American Physical Society under the terms of the Creative Commons Attribution 4.0 International license. Further distribution of this work must maintain attribution to the author(s) and the published article's title, journal citation, and DOI.
Subject Areas: Atomic and Molecular Physics, Condensed Matter Physics, Optics

\section{INTRODUCTION}

The advent of FEL sources operating in the vacuum ultraviolet (VUV) or $\mathrm{x}$-ray spectral region has paved the way for time resolution and for the observation of nonlinear 
and ultrafast processes in the study of the interaction of radiation with matter involving shallow and deep-core electron levels [1-7]. The goal of FELs, in general, is to provide intense and short light pulses with high spectral resolution. Experiments performed at seeded FELs also have the major advantage of the stability of the main pulse parameters such as intensity, duration, time of arrival, and bandwidth. The externally seeded FEL source FERMI, which covers the wavelength range $4-100 \mathrm{~nm}[8,9]$, is at the cutting edge of this new research field [10-16]. Along with the energy per pulse, the FEL pulse duration defines the rate of deposited energy on the sample, which drives the onset of nonlinear processes [17] and the modification of absorption $[1,18]$ during sample illumination.

In structural studies, the aim is to shorten the pulse to the point that it outruns radiation damage and allows (also by means of high intensity) the collection of single-shot diffraction images from single small particles with high spatial resolution $[3,19,20]$. The time structure of the pulse is an extremely relevant parameter in the study of transient properties in solids [21-25], magnetic properties [26,27], and structural phase transition [28], as well as in femtochemistry studies [7] and, in general, in the study of all ultrafast dynamic processes [29-31]. Therefore, accurate control and knowledge of the pulse duration are two of the most important prerequisites for the FEL user. The goal of reducing the pulse duration is intimately connected with interesting machine physics studies.

In recent years, several indirect [32-35] and direct [36-43] methods have been developed to provide reliable temporal profiles, both on average and on a single-shot basis. Indirect methods extract the pulse information from some machine parameters such as longitudinal electron phase space [32,33], fluctuation of the radiated spectrum [34], and number of observed spikes [35]. These parameters are linked to the pulse duration by means of some theoretical models of FEL emission. Since indirect methods extract the FEL pulse temporal information by modeling the FEL output, their application requires calibration by some other approach. On the other hand, methods such as extreme ultraviolet (XUV) autocorrelation in the gas phase [36], cross-correlation in the solid state $[37,38]$ or in the gas phase $[41,44-46]$, and optical laser-driven Terahertz streaking [39] provide temporal information directly by means of the response of an ultrafast physical process to FEL radiation. Most of these studies have been performed on self-amplified spontaneous emission (SASE) FEL sources.

In contrast, FERMI $[9,47]$ is an externally seeded FEL source, based on the high-gain harmonic generation scheme (HGHG) [48]. The FEL process is divided into three steps (see Fig. 1), each occurring in a dedicated structure along the electron beam path: (1) Within a first undulator (the modulator), a UV laser pulse seeds the electron bunch by imprinting the desired temporal properties (intensity profile, phase, and coherence) on its energy distribution; (2) a magnetic chicane (dispersive section) converts the energy modulation into a density modulation at the seed wavelength $\lambda_{0}$ and its higher harmonics; (3) within an undulator array, tuned to a specific harmonic $n$, light emission and amplification occur. The output FEL pulse distribution is the result of the evolution through these steps. With a sufficiently smooth longitudinal electron beam distribution, the pulse properties still mirror those of the seed, and understanding the effect of the three steps gives extra flexibility to predict and control the final pulse temporal profile. The correlation between FERMI pulse properties and seed pulse properties is well-established [49]. A single-shot spectrotemporal characterization of the seeded FERMI pulses was also carried out in a specific double-pulse configuration $[43,50]$ by means of the SPIDER technique [51]; this method allows one to reconstruct the amplitude and phase of the pulses, but it imposes a series of constraints on the FEL conditions of operation, thus limiting its application to a specific machine setup. In this paper, we present the first extended study of the FEL pulse temporal properties from a seeded FEL. The study is based on two cross-correlation techniques, aimed at determining the temporal pulse profile of the externally seeded source FERMI in a wide spectral range and for different machine settings. The first cross-correlation between the FEL and an external laser in the gas phase is referred to as method A, and the second method, where the crosscorrelation takes place in the solid target, is referred to as method B. Several parameters known to affect the pulse

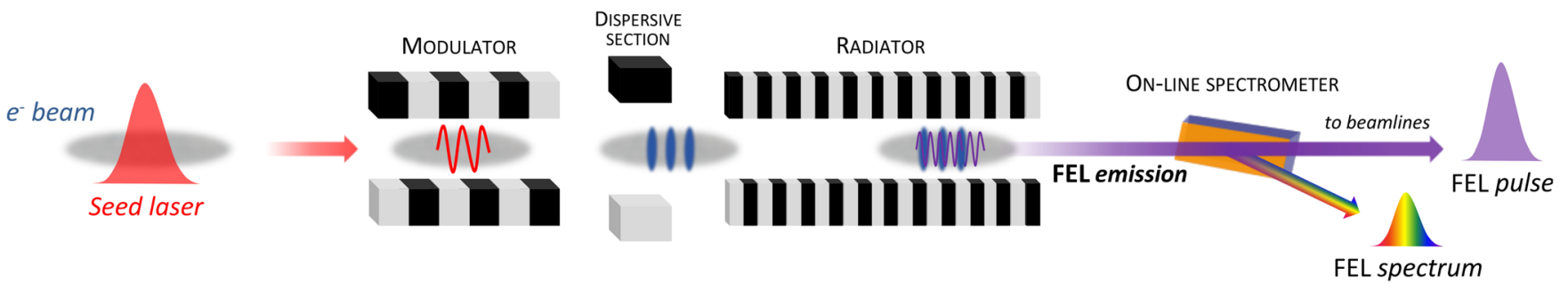

FIG. 1. Layout of a HGHG FEL: UV seed and electrons are superimposed in a first modulator. The UV laser pulse imprints its temporal properties on the electron energy distribution; a dispersive section converts the energy modulation into a density modulation at the seed wavelength $\lambda_{0}$ and at its higher harmonics; the radiator, with resonance tuned to a specific harmonic $n$, amplifies this harmonic. 
duration were varied during the course of these experiments, such as the seed pulse length and the FEL saturation conditions. These sets of measurements therefore represent a case study of the properties of HGHG seeded FEL pulses, as well as a benchmark of the two methods for measuring the temporal pulse shape used. The paper is organized as follows: Section II provides a theoretical model to predict the seeded FEL pulse duration, Sec. III presents the experimental details of methods A and B, and Sec. IV reports the results of both. In Sec. V, we summarize the results and the conclusions supported by the theory.

\section{PULSE DURATION OF A SEEDED FEL}

In a seeded HGHG FEL, the process of transfer of amplitude and phase information of the seed to the electron beam phase space and the following FEL harmonic amplification are equally influenced by the properties of the electron beam and of the seed laser pulse [49]. Any longitudinal variation of the FEL resonance condition and of the FEL coupling parameters - such as the beam current, the quality of the longitudinal and transverse phase space, the electron energy distribution, and the phase chirp of the seed pulse- has an influence on the final pulse shape and duration [52]. The studies presented here were performed in a mode of operation where the electron bunch was much longer than the seed pulse and the electron beam properties were sufficiently homogeneous; thus, the amplification process may take place in a longitudinal region longer than the seed pulse itself. These settings are standard for the setup and tuning of a single-stage seeded source such as FERMI FEL-1, which is operated in a regime where pulse length and pulse structure are largely determined by the seed properties. However, even in these ideal conditions, all the intermediate processes-e.g., conversion of the seed into electron-beam energy modulation, frequency up-shift, and finally the amplification-play an important role in the determination of the output-pulse length and structure. The FEL saturation mechanism also has a profound effect on the output-pulse properties: The dynamics of electrons trapped in the ponderomotive potential associated with the laser field induces amplitude and phase modulations of the FEL output pulse. In deeply saturated conditions, multiple pulses may appear [53], and the FEL may enter a nonlinear dynamical regime where the FEL pulse properties are almost independent of the seed pulse [54-57].

We now go through the steps of energy modulation and harmonic conversion (Sec. II A) and final amplification (Sec. II B). We relate the properties of the FEL pulse to those of the seed (electric field envelope $E_{L}$, wavelength $\lambda_{0}$ ), electron beam (energy $\mathcal{E}$, energy spread $\sigma_{\gamma}$, longitudinal charge density $\rho_{\mathrm{e}}$, bunching factor $b_{n}$ ), undulator (period $\lambda_{u}$, number of periods $N$, overall length $N \lambda_{\mathrm{u}}$, gain length $L_{g}$ ), and dispersive section (strength $R_{56}$ ). We further indicate the speed of light in vacuum, $c$, the electron rest mass $m_{0}$, and the electron relativistic factor $\gamma=\mathcal{E} / m_{0} c^{2}$.

\section{A. Beam energy-density modulation}

The harmonic conversion process starts in a first undulator (modulator) where the ultrarelativistic electron beam is modulated in energy via the interaction with an intense seed laser pulse. The relative energy modulation depth is proportional to the laser electric field $E_{L}$,

$$
\frac{\Delta \gamma}{\gamma} \propto E_{L}
$$

In order to initiate lasing, $\Delta \gamma / \gamma$ has to be larger than the relative natural beam energy spread $\sigma_{\gamma}$. Any high-gain FEL amplifier has a limited gain bandwidth of the order of $\sigma_{\omega} \simeq \rho_{\mathrm{FEL}}$, associated with the slippage of the radiation field over the electron beam. The parameter $\rho_{\text {FEL }}$ [58] defines the gain length $L_{\mathrm{g}}$; hence, the exponential growth rate of the amplifier is $P(z)=\exp \left(z / L_{\mathrm{g}}\right)$, where $L_{g}=$ $\lambda_{\mathrm{u}} /\left(4 \pi \sqrt{3} \rho_{\mathrm{FEL}}\right)[59,60]$ and $z$ is the distance traveled by the beam along the undulator. When the undulator length $N \lambda_{\mathrm{u}}$ is shorter than the gain length $L_{\mathrm{g}}$, as in the modulator, the gain bandwidth scales as $\sigma_{\omega} \approx 1 / 2 N$. In the following, we assume that the seed pulse has a limited bandwidth, smaller than the bandwidth of the modulator and, after harmonic conversion, of the amplifier. Under such conditions, the effects of slippage of the field on the electrons can be neglected, and the energy modulation imprinted on the electron phase space carries all the amplitude and phase information of the seed pulse. The seed laser pulse plays no additional role after the interaction with the electron beam in the modulator. The source of the radiation emission in the final FEL amplifier is the charge density modulation, expressed as a "bunching factor," i.e., as the Fourier coefficient of the electron density modulation at the resonant frequency of the amplifier. In order to convert the energy modulation induced by the seed into a density modulation, the beam traverses a dispersive section: a magnetic device where the electron path length is inversely proportional to the electron energy. Two electrons whose energies differ by $\delta \gamma$ will be longitudinally shifted by

$$
\delta \zeta=\frac{\delta \gamma}{\gamma} R_{56}
$$

where $\zeta=z-\beta_{z}$ ct is the longitudinal coordinate in the moving electron beam frame [61] and where the momentum compaction $R_{56}$ represents the "strength" of the dispersive section. This results in a modulation of the longitudinal charge density of the electron beam $\rho_{e}(\zeta)$, containing Fourier components at the seed laser frequency and at the higher-order integer harmonics. The bunching factor at the $n$th harmonic of the seed and at position $\zeta$ is defined as 


$$
b_{n}(\zeta)=\frac{1}{\lambda_{0}} \int_{\zeta}^{\zeta+\lambda_{0}} \rho_{e}\left(\zeta^{\prime}\right) e^{-2 \pi i n \zeta^{\prime} / \lambda_{0}} d \zeta^{\prime}
$$

It can be shown that $b_{n}$ depends on the initial energy spread $\sigma_{\gamma}$, on the dispersion $R_{56}$ appearing in Eq. (2), and on the energy modulation $\Delta \gamma$, which, under our assumptions, is the only factor introducing a dependence on $\zeta$. The amplitude of $b_{n}$ can also be written as [48]

$$
\left|b_{n}(\zeta)\right|=\exp \left[-\frac{1}{2}\left(\frac{2 \pi n}{\lambda_{0}} \sigma_{\gamma} R_{56}\right)^{2}\right] J_{n}\left(\frac{2 \pi n}{\lambda_{0}} \frac{\Delta \gamma}{\gamma} R_{56}\right),
$$

where $J_{n}$ is a Bessel function of the first kind of order $n$, and we define the modulation-dispersion product

$$
\chi_{n}\left(\Delta \gamma, R_{56}\right)=\frac{2 \pi n}{\lambda_{0}} \frac{\Delta \gamma}{\gamma} R_{56} .
$$

In summary, the density modulation is the source term for the field growth in the final amplifier. This parameter should be optimized by tuning the dispersion $R_{56}$ and the energy modulation $\Delta \gamma / \gamma$ while keeping the latter as low as possible. The energy modulation is linearly proportional to the electric field of the seed laser [Eq. (1)], and at a given dispersion and harmonic order $n$, the expression in Eq. (4) relates the longitudinal amplitude of the seed electric field $E_{L}(\zeta)$ to the bunching factor $b_{n}(\zeta)$ that describes the longitudinal electron beam profile.

In the first two gain lengths within the amplifier, the field grows approximately as

$$
E_{\mathrm{FEL}}(\zeta, z) \propto z b_{n}(\zeta)
$$

After these two gain lengths, a radiation pulse up-shifted in frequency to the resonant frequency of the undulator, with the longitudinal phase and amplitude profiles of the bunching factor $b_{n}(\zeta)$, will be exponentially amplified. In the limit of negligible slippage of the radiation pulse over the electron bunch with respect to the converted pulse duration, the pulse properties will be preserved during amplification. Therefore, we have

$$
E_{\mathrm{FEL}}(\zeta, z)=E_{\mathrm{FEL}}\left(\zeta, 2 L_{\mathrm{g}}\right) \exp \left(z / 2 L_{\mathrm{g}}-1\right),
$$

and the longitudinal pulse intensity profile at the exit of the amplifier will be proportional to $b_{n}^{2}(\zeta)$. Let us now analyze Eq. (4) in more detail under these assumptions, which we will then relax to explore the case where saturation may occur at the end of the amplifier. If we assume that our seed pulse has a Gaussian intensity shape with rms intensity duration $\sigma_{\zeta}$,

$$
E_{\mathrm{L}}(\zeta)=E_{\mathrm{L}}(0) \exp \left(-\frac{\zeta^{2}}{4 \sigma_{\zeta}^{2}}\right)
$$

and that the peak value $E_{\mathrm{L}}(0)$ corresponds to a modulation $\Delta \gamma$, we may introduce a dependence of the Bessel function argument on the coordinate $\zeta$ via the longitudinal laser intensity profile, following the method used in Ref. [62]. The longitudinal intensity distribution of the FEL pulse is therefore

$\left|E_{\mathrm{FEL}}\left(n, \zeta, \chi_{n}\right)\right|^{2} \propto J_{n}\left[\chi_{n}\left(\Delta \gamma, R_{56}\right) \exp \left(-\frac{\zeta^{2}}{4 \sigma_{\zeta}^{2}}\right)\right]^{2}$.

Under conditions of low seed power and low dispersion $\left[\chi_{n}\left(\Delta \gamma, R_{56}\right) \ll 1\right]$, we may expand the Bessel function around zero; thus, at the lowest order, we get

$$
E_{\mathrm{FEL}}(n, \zeta) \propto \exp \left(-\frac{n \zeta^{2}}{4 \sigma_{\zeta}^{2}}\right)
$$

i.e., the FEL radiation pulse maps the seed pulse profile, and the rms distribution scales as the square root of the harmonic order $\sigma_{\zeta}^{\mathrm{FEL}}=\sigma_{\zeta} / \sqrt{n}$.

The conditions analyzed so far are rather relaxed in terms of attainable intensity of the FEL pulse; indeed, one generally considers, as optimized conditions for FEL emission, those for which the bunching factor is maximized at a given modulation $\Delta \gamma$; i.e., the Bessel function in Eq. (4) is maximized. The Bessel function of $n$th order reaches the first local maximum when its argument is approximately [63]

$$
\chi_{n}\left(\Delta \gamma, R_{56}\right)=\chi_{n}^{\max }=n\left(1+\sqrt{\frac{2}{3}} n^{-\frac{2}{3}}\right) .
$$

In Fig. 2 (right panel), the optimal situation at the 10th harmonic, providing the highest bunching at the peak seed laser intensity (b, solid line), is compared to two detuned cases, corresponding to a $20 \%$ reduction (a, dotted line) or increase (c, dashed line) of the modulation-dispersion product $\chi_{n}$. A relatively modest change in the settings of the dispersion or of the seed laser intensity has important effects on the output-pulse profile. In the overbunched case

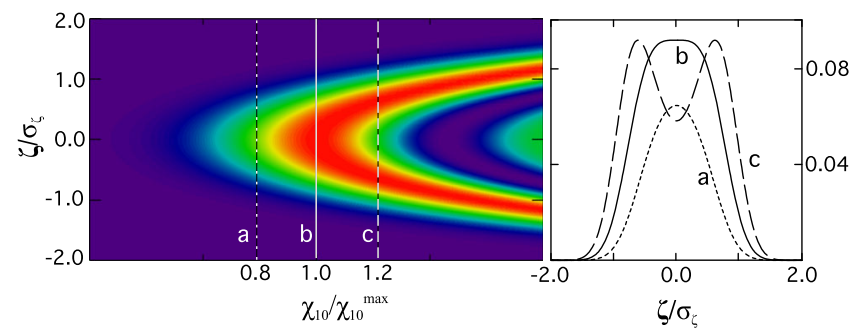

FIG. 2. Pulse longitudinal profile [Eq. (9), with $n=10$ ] as a function of the normalized longitudinal coordinate $\zeta / \sigma_{\zeta}$ and of the modulation-dispersion product (left panel). Pulse longitudinal profiles (right panel) at selected $\chi_{10}$ positions. The dotted line (a) shows $\chi_{10}=0.8 \chi_{10}^{\max }$, the solid line (b), $\chi_{10}=\chi_{10}^{\max }$, and the dashed line (c), $\chi_{n}=1.2 \chi_{10}^{\max }$. 
(c, dashed line), the pulse splits into two sub-bunches, a well-known technique used to generate multiple pulses for pump and probe experiments $[53,66,67]$. When the pulse splits, shortening of the subpulses is associated with the spectral broadening, and spectral structures are related to interferences between the two (or more) subpulses $[49,68]$ or, in the case of a chirped seed, to a spectral mapping of the temporal distribution $[49,69]$.

In order to quantify the evolution of the FEL pulse duration, one can calculate the rms length of the intensity distribution $\left|E_{\mathrm{FEL}}\left(n, \zeta, \chi_{n}\right)\right|^{2}$ as

$$
\sigma_{\zeta}^{\mathrm{FEL}}\left(n, \chi_{n}\right)=\sqrt{\frac{\int_{-\infty}^{+\infty}\left|E_{\mathrm{FEL}}\left(n, \zeta, \chi_{n}\right)\right|^{2} \zeta^{2} \mathrm{~d} \zeta}{\int_{-\infty}^{+\infty}\left|E_{\mathrm{FEL}}\left(n, \zeta, \chi_{n}\right)\right|^{2} \mathrm{~d} \zeta}},
$$

where we have exploited the fact that the intensity distribution is a symmetric function of $\zeta$, i.e., $\int_{-\infty}^{+\infty}\left|E_{\mathrm{FEL}}\left(n, \zeta, \chi_{n}\right)\right|^{2} \zeta d \zeta=0$.

In Fig. 3, the dependence of the pulse length on the harmonic conversion order is shown. The continuous black line represents the rms length of the FEL intensity distribution normalized to the seed pulse duration $\sigma_{\zeta}$ and calculated at $\left(\chi_{n}=\chi_{n}^{\max }\right)$.

The pulse length at the maximum of the Bessel function scales approximately as (Fig. 3, blue dashed line):

$$
\sigma_{\zeta}^{\mathrm{FEL}}\left(n, \chi_{n}^{\max }\right) \simeq \frac{\sigma_{\zeta}^{\mathrm{FEL}}\left(1, \chi_{n}^{\max }\right)}{n^{1 / 3}} \sim \frac{7}{6} \frac{\sigma_{\zeta}}{n^{1 / 3}} .
$$

This behavior is compared with the function $\sigma_{\zeta}^{\mathrm{FEL}}\left(n, \chi_{n}\right)$ calculated at $\chi_{n}=0.5 \chi_{n}^{\max }$ (black dotted line) and $\chi_{n}=$ $1.1 \chi_{n}^{\max }$ (black dashed line). An excess of $10 \%$ in the seed electric field amplitude ( $20 \%$ in intensity) or in the

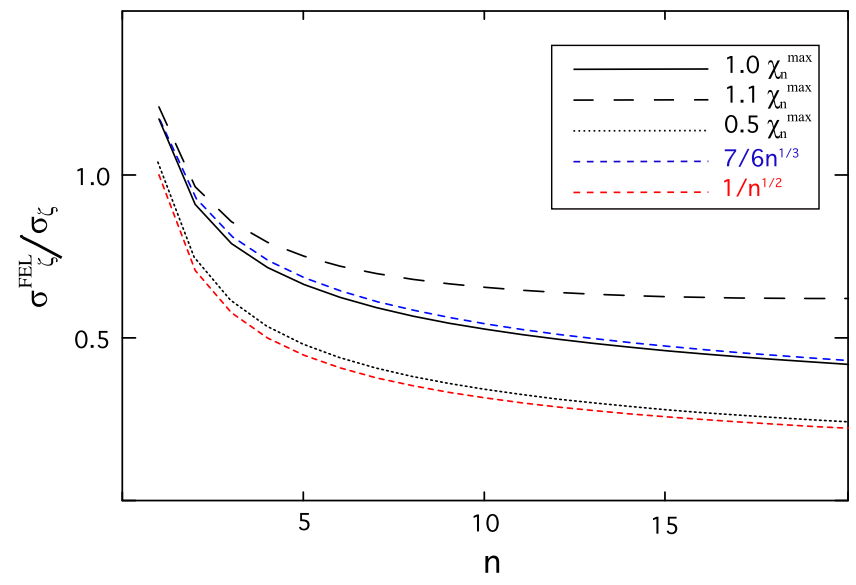

FIG. 3. Relative pulse length $\sigma_{\zeta}^{\mathrm{FEL}}\left(n, \chi_{n}\right) / \sigma_{\zeta}$, as a function of the harmonic conversion order $n$. Solid black line: $\chi_{n}=\chi_{n}^{\max }$. Black dotted line: $\chi_{n}=0.5 \chi_{n}^{\max }$. Black dashed line: $\chi_{n}=1.1 \chi_{n}^{\max }$. Red dashed line: Solution in the limit $\chi_{n} \ll \chi_{n}^{\max }$, i.e., the function $\sigma_{\zeta}^{\mathrm{FEL}} / \sigma_{\zeta}=1 / \sqrt{n}$. Blue dashed line: The function $7 /\left(6 n^{1 / 3}\right)$.

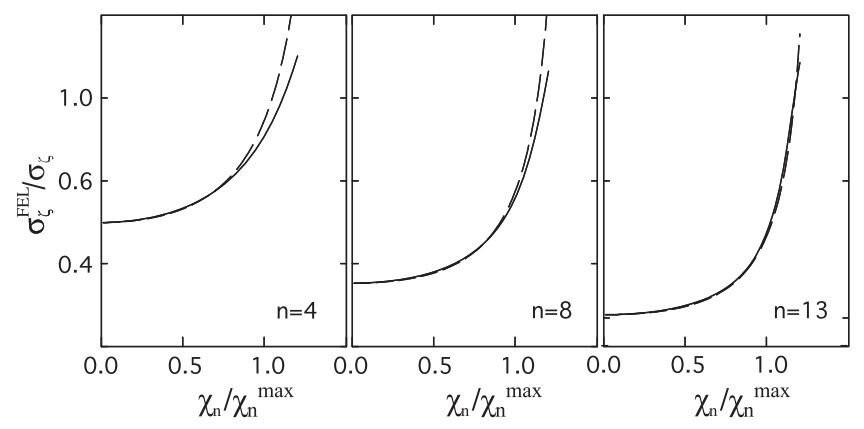

FIG. 4. Pulse length vs the function $\chi_{n}\left(\Delta \gamma, R_{56}\right)$ for three different harmonics: $n=4$ (left panel), $n=8$ (center panel), and $n=13$ (right panel). Dashed line: The approximating function, Eq. (14).

dispersive strength leads to a substantial growth of the pulse length, especially at high harmonic orders. On the other hand, halving the dispersion or the seed field amplitude leads to a condition well approximated by the solution in the limit $\chi_{n} \ll \chi_{n}^{\max }$, i.e., $\sigma_{\zeta}^{\mathrm{FEL}}=\sigma_{\zeta} / \sqrt{n}$ (red dashed line).

In Fig. 4, we show the growth of the pulse duration associated with an increase of the factor $\chi_{n}\left(\Delta \gamma, R_{56}\right)$ for three different harmonics (solid line). For comparison, the dashed line represents the corresponding analytical functions

$$
d\left(n, \chi_{n}\right)=\frac{1}{\sqrt{n}}\left\{1-\frac{2}{9} \chi_{n}^{2}\left[1+\left(1-\frac{2}{n}\right) \chi_{n}^{2}\right]\right\}^{-1}
$$

empirically derived by approximating the pulse width from Eq. (12) and providing a reasonable approximation for the pulse length increase in the ranges $1 \leq n \leq 20$ and $0<\chi_{n}<1.2 \chi_{n}^{\max }$.

\section{B. Amplification and saturation effects in the amplifier}

An excessive bunching factor in Eq. (7) can bring the FEL peak power close to the saturation level $P_{\max } \sim$ $1.6 \rho_{\mathrm{FEL}} P_{\mathrm{e}}$ [70-72], with $P_{\mathrm{e}}$ the power carried by the electron beam. Saturation arises from a combination of gain depression induced by an increased e-beam energy spread, and of phase mismatch between the bunched beam and the copropagating amplified optical wave due to the energy detuning associated with the electron kinetic energy transfer to the optical field. A pulse splitting process, analogous to the one after the modulator and dispersive section, takes place at the onset of saturation, where the exponential growth of the power is suppressed at the pulse peak position but may continue on the leading and trailing edges of the pulse where the field amplitude is lower. This causes a further increase of the pulse length, typically accompanied by spectral broadening and by the appearance of structures in the spectrum indicating the formation of a correlated structure in the temporal profile. Another effect associated with the different propagation velocity of the 
electron beam and the optical field (slippage) is the growth of an asymmetry between the front pulse, shifting over a fresh portion of the electron beam and the rear pulse, that conversely interacts with electrons heated by the tail of the front pulse. At the longest wavelength at FERMI, the slippage distance over the entire amplifier undulator is of the order of $30 \mathrm{fs}$. We therefore expect this effect to be modest at high harmonic orders and when the seed pulse duration is comparable to, or longer than, $100 \mathrm{fs}$. Under normal conditions, the FEL parameters are tuned to reach the onset of saturation just at the end of the amplifier to minimize the saturation effects discussed in Refs. [53,56]. In Fig. 5, we show the behavior of the pulse lengthening $\sigma_{\zeta}^{\mathrm{FEL}-\mathrm{sat}} / \sigma_{\zeta}^{\mathrm{FEL}}$ vs $z$ along the undulator. The plot is derived from the analytical model of the HGHG process in Ref. [64], under the assumption that seed intensity and dispersion are tuned to reach the threshold $0.5 P_{\max }$ at the position $z=z_{\text {sat }}$. If $z_{\text {sat }}$ corresponds to the end of the radiator of length $\left(z_{\mathrm{sat}}=L_{\mathrm{u}}\right)$, we have a pulse lengthening of about $10 \%$ associated with saturation. A marginal pulse shortening at the cost of a drop in the pulse peak power may be obtained by reducing the bunching factor at the entrance of the amplifier, which shifts the position $z_{\text {sat }}$ forward. Conversely, if we increase the bunching factor to have $z_{\mathrm{sat}}<L_{\mathrm{u}}$, an increase of peak power and pulse energy comes at the cost of a further increase of the pulse duration.

In summary, we have analyzed independently the two phases mostly influencing the temporal properties of a seeded FEL: (1) the density modulation or harmonic conversion phase and (2) the amplification or saturation phase in the amplifier. Analogous to the harmonic conversion in optical nonlinear processes, in phase (1) we have a shortening of the pulse scaling with the harmonic order as $1 / \sqrt{n}$ (Fig. 2, $\chi_{n} / \chi_{n}^{\max }<0.8$, position a). Optimized tuning aimed at maximizing the bunching factor for a given induced energy modulation $\Delta \gamma\left(\chi_{n} / \chi_{n}^{\max } \approx 1\right)$

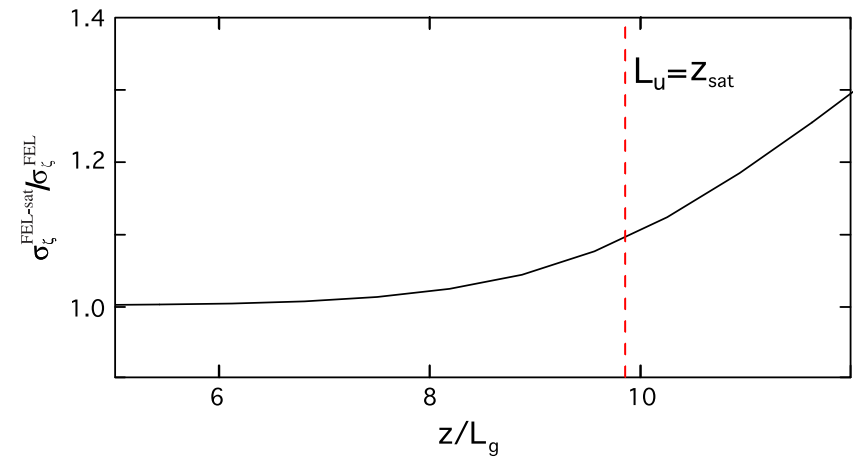

FIG. 5. Increase of the pulse duration associated with saturation effects at the end of the radiator. The behavior is independent of the harmonic order if the abscissa is scaled by the FEL gain length $L_{g}$. The vertical line indicates the condition of undulator length equal to the saturation length $L_{u}=z_{\text {sat }}$ occurring after approximately 10 gain lengths in this example. leads to a pulse duration scaling with the harmonic order as $7 /\left(6 n^{1 / 3}\right)$ (Fig. 2, position b). A further increase of the parameter $\chi_{n}$ leads to pulse splitting (Fig. 2, position c). In phase (2), the amplification process, with the hypothesis of uniform electron beam parameters and narrow bandwidth of the FEL pulse, leaves the pulse profile almost unchanged until saturation is reached. Saturation is first reached at the pulse peak and then at the pulse wings. This leads to a second pulse lengthening (and successive splitting), analogous to the one that occurred in step (1). The excess of a bunching factor at the entrance of the amplifier expedites the onset of saturation and lengthening. These structural changes of the pulse shape are always associated with an increase of the parameter $\chi_{n}$ containing the product of the field amplitude of the seed and the strength of the dispersion. We therefore analyze the experimental data in relation to relative variations of parameter $\chi_{n}$, distinguishing a short pulse regime, a maximum power regime, and a saturated regime.

\section{EXPERIMENTAL METHODS AND SETUPS}

FERMI is composed of two independent FELs that share the same electron source and photon transport optics. The two sources are identified as FERMI FEL-1 and FERMI FEL-2 (for brevity, FEL-1 and FEL-2, respectively). FEL-1 consists of a single HGHG stage and covers the range 20-100 nm [8]. FEL-2 consists of a double HGHG stage: The first is a shorter replica of FEL-1 whose pulses are used to seed the second stage to produce radiation in the 4-20$\mathrm{nm}$ range. Under optimized conditions, the pulse has a typical energy ranging from a few to hundreds of $\mu \mathrm{J}$, with fluctuations that can be as low as 5\% (rms, on FEL-1), and a single-mode spectral distribution with Gaussian shape [73]. The full wavelength tunability of FERMI derives from the broad tunability of the seed laser via an optical parametric amplifier (OPA) module and the use of variable gap undulators. The data presented here were collected at the low photon energy FEL-1 and at the first stage of the FEL-2 (indicated as FEL-2.1) sources. FEL-1 delivers photons directly to the end user. FEL-2.1 seeds the second short-wavelength stage of FEL-2, and the pulse properties measured at this intermediate stage provide information on the final pulse properties of FEL-2. Measurements were also done with the FEL operating with the seed generated in two different conditions: as the third harmonic of the Ti: Sapphire laser amplifier (which will be indicated as THG) and as the third harmonic of a wavelength-tunable OPA.

All the optics of the FERMI beam lines (see Fig. 6 and its caption) are reflective, and because of the intrinsic narrow bandwidth of the FEL spectrum, the optical transport system should not affect the FEL pulse length.

Two different experimental setups for the determination of the pulse duration were utilized. One was installed at the low-density matter (LDM) beam line [78] and the other at the diffraction and projection imaging beam line (DiProI) 


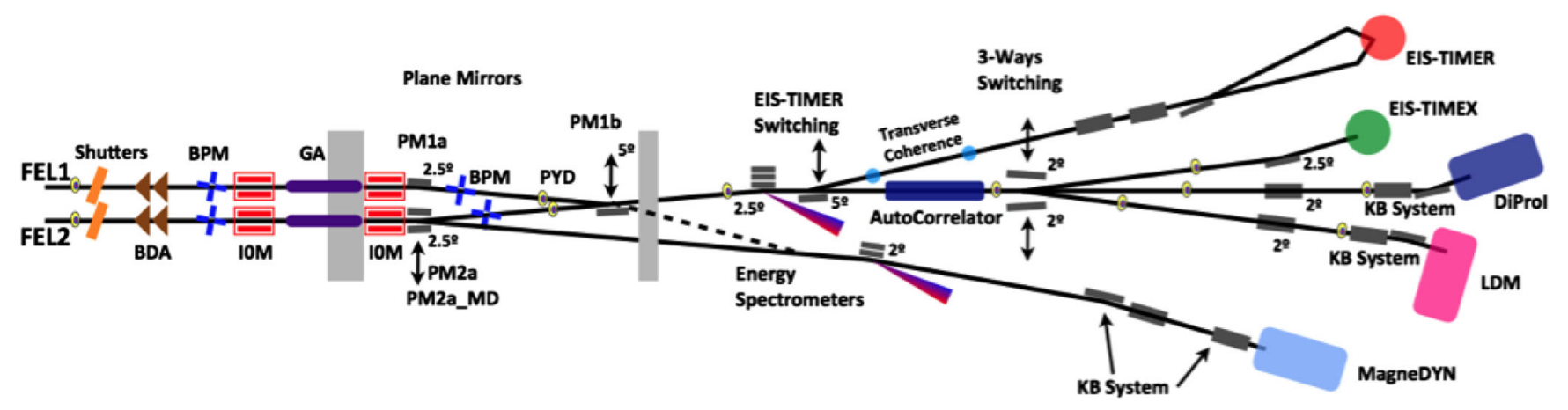

FIG. 6. Layout of the FERMI beam transport section. The DiProI and LDM experimental stations share a common photon transport system that includes a beam-defining aperture (BDA), photon beam position monitors (BPM), a gas cell monitoring the FEL fluency (IOM), a gas attenuator (GA), a solid-state photon yield detector (PYD), an energy spectrometer, an autocorrelator, and plane steering mirrors [74-76]. On LDM and DiProI, the FEL beam is focused by a system of KB mirrors [77].

$[79,80]$. The experimental techniques adopted on LDM and DiProI are both based on cross-correlation between the FEL pulse and an external laser pulse. This source is the same Ti:Sapphire laser generating the seed for the HGHG process. This solution provides an extremely low jitter between the FEL pulse and the Ti:Sapphire external laser (about $6 \mathrm{fs}$ ) and is commonly adopted at FERMI for pump and probe experiments $[81,82]$. On the LDM beam line, the pulse duration was measured by monitoring the intensity of sidebands in the photoelectron spectrum of helium $[44,83,84]$ as a function of the time delay $\Delta t$ between the FEL pump pulse and an IR probe pulse [44-46]. On the DiProI beam line, the cross-correlation was obtained by monitoring the transient transmission change of visible light in a $\mathrm{Si}_{3} \mathrm{~N}_{4}$ membrane induced by the FEL pulse [37] in the so-called tilted-front geometry. The LDM experiment is a direct cross-correlation measurement, but it requires scanning the temporal delay between the FEL and the external laser. The DiProI experiment is more elaborate, but it allows single-shot measurements; in principle, the underlying method could be used as an online diagnostic in the case of experiments involving transmissive targets. More details about each method and experimental setup are provided in Secs. III A and III B, respectively.

\section{A. Two-color photoemission cross-correlation}

The physical mechanism behind the FEL-IR crosscorrelation signal is the appearance of IR-induced sidebands in a photoelectron spectrum. In our experiment, using a helium gas jet, a single FEL photon promotes one He electron above the ionization potential, giving a photoemission line at energy $E_{0}$; simultaneous absorption or stimulated emission of one or more IR photons transfers intensity from this main line into satellite peaks, called sidebands, at energies $E_{0} \pm m h \nu_{\mathrm{IR}}$ (Fig. 7), where $h \nu_{\mathrm{IR}}$ is the IR photon energy and $m$ is the sideband order (below, we indicate the sidebands at orders $1,2, \ldots, m$ as $\mathrm{SB} 1, \mathrm{SB} 2, \ldots, \mathrm{SB} m)$. The measurements were done in linear horizontal polarization to avoid dichroic effects [11]. In the perturbative regime, the intensity of the $m$ th sideband, $I_{\mathrm{SB} m}$, as a function of the delay $\Delta t$ between the IR and FEL pulse is proportional to the $m$ th power of the IR intensity $I_{\mathrm{IR}}[46]$,

$$
I_{\mathrm{SB} m}(\Delta t) \propto \int_{-\infty}^{\infty} I_{\mathrm{IR}}(t-\Delta t)^{m} I_{\mathrm{FEL}}(t) d t .
$$

Given the time structure of the IR pulse, the FEL pulse can be deconvolved from the cross-correlation curve $I_{\mathrm{SB} m}(\Delta t)$. For Gaussian temporal profiles of the two lasers, $I_{\mathrm{SB} m}(\Delta t)$ is also a Gaussian, and Eq. (15) yields a relation between the full width at half maximum (FWHM) of the cross-correlation curve, $\tau_{C}$, and those of the FEL and IR pulses $\left(\tau_{\mathrm{FEL}}\right.$ and $\tau_{\mathrm{IR}}$, respectively) [46,85]:

$$
\tau_{C}=\sqrt{\tau_{\mathrm{FEL}}^{2}+\frac{\tau_{\mathrm{IR}}^{2}}{m}} .
$$

Equation (16) shows that the effective IR temporal width scales with $m$ as $1 / \sqrt{m}$. Therefore, within the limit of the perturbative regime, by increasing $m, \tau_{C}$ is bound to converge to $\tau_{\mathrm{FEL}}$ [86].

Note that, in the general case, the FWHM of the crosscorrelation is also broadened by the temporal jitter between the IR and the FEL [41,46,85], appearing on the right side of Eq. (16) as an additional term $\tau_{J}$ to be added in quadrature. As already mentioned, this term is not relevant in our case because of the modest arrival-time jitter compared to the typical pulse length. This was explicitly verified by monitoring over time $\left(1.5 \times 10^{4}\right.$ shots $)$ the intensity of the sideband SB2 at the point of maximum derivative of the cross-correlation curve. At this point of the curve, a potential time jitter causes the maximum fluctuation in the signal on top of the statistical noise. After deconvolution of the statistical noise (estimated by measuring, over the same amount of time, the sideband intensity 
at the flat top of the cross-correlation curve), the sideband signal fluctuation was found to be consistent with the jitter estimate $\tau_{J} \lesssim 5 \mathrm{fs}$. The contribution of the jitter is therefore negligible in the measurements of pulse durations typically 1 order of magnitude larger.

At high IR intensity, sidebands approach saturation, and the simple power law $I_{\mathrm{SB} m} \propto I_{\mathrm{IR}}^{m}$ loses validity; in practice, one replaces the exponent $m$ with an effective value $\alpha<m$, which increases the effective temporal width of the IR pulse that enters Eq. (16). The value of $\alpha$ (dependent on $m$ and the range of $I_{\mathrm{IR}}$ ) can be empirically determined $[46,85]$. When saturation occurs, use of the perturbative limit $\alpha=m$ amounts to an overestimate of the deconvolved pulse duration [see Eq. (16)]. Because higher-order sidebands are harder to saturate [87], we expect that with increasing $m$, the validity of the perturbative regime equation holds for higher IR intensities.

\section{Experimental setup}

The FEL-1 source was operated in the OPA seeding mode, while FEL-2.1 was operated in the THG seeding mode at a wavelength of $261.1 \mathrm{~nm}$. In both cases, the FEL intensity was about $5 \times 10^{12} \mathrm{~W} / \mathrm{cm}^{2}$. The seed laser pulse duration was measured via cross-correlation, and in both cases (THG and OPA), the duration was about $140 \mathrm{fs}$ FWHM. The time duration of the second IR pulse was $(90 \pm 1)$ fs $(\mathrm{FWHM})$, as measured by a dedicated, singleshot autocorrelator. The focal spot size of the infrared laser was adapted to completely dress the spot of the FEL beam, as measured on a yttrium aluminum garnet (YAG) screen $(50 \mu \mathrm{m}$ from FEL-1 and $90 \mu \mathrm{m}$ from FEL-2.1). The maximum IR energy per pulse available at the sample was $350 \mu \mathrm{J}$, corresponding to a maximum intensity of about $4 \times 10^{13} \mathrm{~W} / \mathrm{cm}^{2}$ (on a spot of about $100 \mu \mathrm{m}$ ) for FEL-1 and for FEL-2.1; the intensity could be continuously varied from $0 \%$ to $100 \%$ by a crossed-polarizer arrangement. For FEL-1, the intensity used for the measurements was about $3 \times 10^{12} \mathrm{~W} / \mathrm{cm}^{2}$, for FEL-2.1 of about $1 \times 10^{13} \mathrm{~W} / \mathrm{cm}^{2}$.

Photoemission spectra were measured with a custommade magnetic bottle spectrometer called FERMI-FELCO, akin to the FELCO instrument used at the LCLS $[31,88]$. The magnetic bottle is essentially an electron time-of-flight spectrometer (e-TOF) with a static magnetic field shaped to allow collection of electrons over a wide solid angle $(4 \pi$, for FERMI-FELCO, which, after taking into account the detector efficiency, amounts to an overall efficiency of about 50\%). Temporal and spatial overlap between FEL and IR was optimized by means of the sequential two-color photoemission from $\mathrm{He}$ via the $\mathrm{He}_{1 s 5 p}$ resonant state $\mathrm{He}+h \nu_{\mathrm{FEL}} \rightarrow \mathrm{He}_{1 s 5 p}+m h \nu_{\mathrm{IR}} \rightarrow \mathrm{He}^{+}+e^{-}$. For delays between the two lasers $\Delta t$, much smaller than the lifetime of the $1 s 5 p$ state (nanoseconds), this photoemission signal appears as a step function that also allows an accurate determination of the $\Delta t=0$. The IR delay was scanned by a motorized stage in steps of $20 \mathrm{fs}$.

\section{Data recording, calibration, and processing}

Single-shot time-of-flight spectra were recorded and aggregated (typically over about 200 shots for a given delay) by a digitizer with $1 \mathrm{~ns}$ resolution. The area of the sidebands was calculated by numerical integration. The sidebands are well separated, and the choice of integration limits is not critical, except when an intense peak (usually the main band) introduces signal ringing, which may cause signal cross-talk. Since, for absorption sidebands, the less intense ones precede the more intense ones (particularly the main band) in time, with the opposite for emission sidebands, the former, immune from signal cross-talk, were preferred for data analysis. Absorption sidebands are also sharper in time and rise above the background even at low intensity [see Fig. 7(b)]. The time of flight $t$ to kinetic energy $\left(E_{\mathrm{K}}\right)$ calibration was done by fitting reference peaks to the formula $E_{\mathrm{K}}=\left(a /\left(t-t_{0}\right)\right)^{2}+b$. This step was only necessary to confirm peak assignment and does not enter the data analysis. The cross-correlation curve is obtained as a plot of the average area of the chosen sideband versus delay time. Although shot-to-shot analysis is not possible, we also correlated the average pulse length to the average bandwidth of the FEL available on a shot-to-shot basis from the FEL spectrometer PRESTO [89].

In the case of data acquired from FEL-1, at the working IR intensity of about $3 \times 10^{12} \mathrm{~W} / \mathrm{cm}^{2}$, only two sidebands could be measured [Fig. 7(a)]. The pulse duration values are extracted from SB2. Cross-correlation curves from SB1 and SB2 agree within 10\%, which is an indication

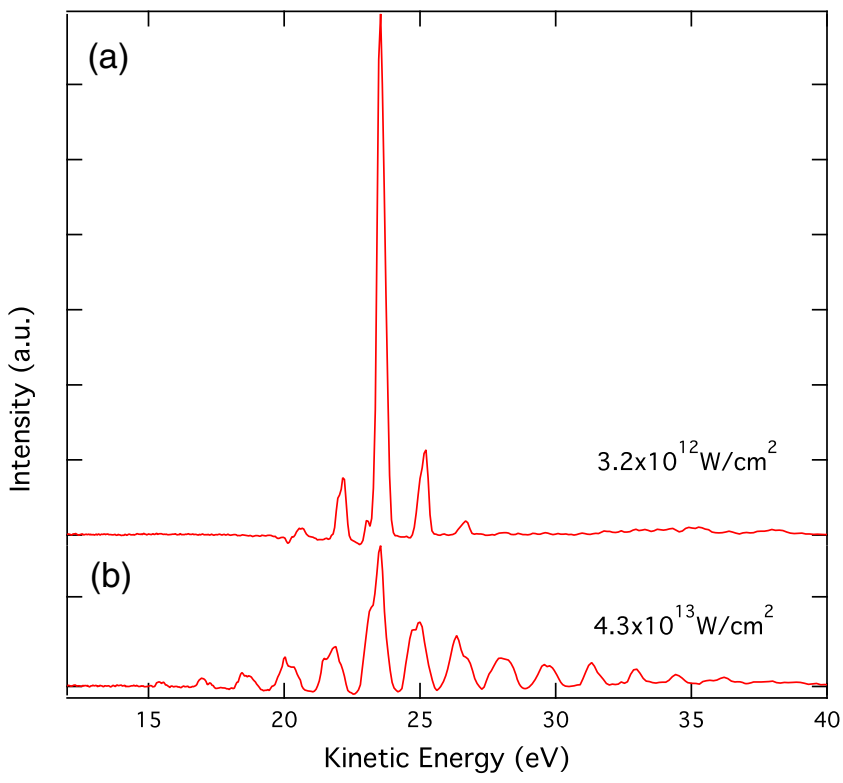

FIG. 7. Two-color (FEL at $25.78 \mathrm{~nm}$, IR at $784 \mathrm{~nm}$ ) photoelectron spectra of atomic He acquired at time delay $\Delta t=0$ for IR intensities: (a) about $3.2 \times 10^{12} \mathrm{~W} / \mathrm{cm}^{2}$ (used for the pulse duration on FEL-1) and (b) about $4.3 \times 10^{13} \mathrm{~W} / \mathrm{cm}^{2}$ (maximum intensity available). 

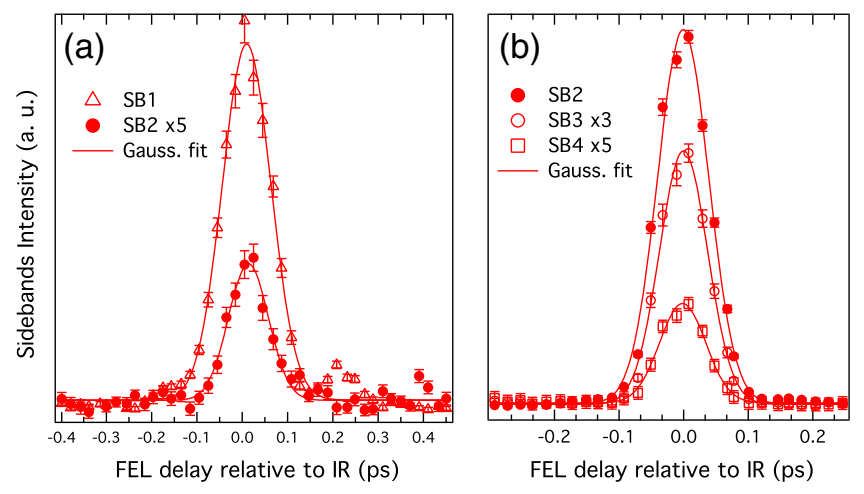

FIG. 8. Cross-correlation curves derived from He sidebands as a function of the delay between the FEL and IR pulses. (a) FEL-1 pulses, IR intensity of about $3.2 \times 10^{12} \mathrm{~W} / \mathrm{cm}^{2}$. (b) FEL-2.1 pulses, IR intensity of about $1.4 \times 10^{13} \mathrm{~W} / \mathrm{cm}^{2}$.

that saturation effects are not dominant in these conditions, although the systematically longer value of the pulse duration obtained from SB1 indicates the onset of saturation for this sideband. In the case of data acquired from FEL-2.1, the IR intensity was higher, about $1 \times 10^{13} \mathrm{~W} / \mathrm{cm}^{2}$, and sidebands up to the fifth order could be measured. In this case, the trend in the FEL pulse duration versus sideband order indicates saturation for SB1 and SB2; conversely, the pulse duration values from SB3 and SB4 are consistent within 4\%; SB5, although measurable, was discarded because of a poorer signal-to-noise ratio. As an example, consider the crosscorrelation curves of Fig. 8: For FEL-1 [Fig. 8(a)], the deconvolution via Eq. (16) yields a pulse duration measurement of $(93 \pm 3)$ fs from SB1 and $(85 \pm 4)$ fs from SB2; for FEL-2.1 [Fig. 8(b)], the deconvolution yields a pulse duration of $((77 \pm 3)$ fs for SB2, $(68 \pm 1)$ fs for SB3, and $(70 \pm 2)$ fs for sideband 4 . In summary, the measurements reported for FEL-2.1 are from SB3 (better signal-tonoise ratio in comparison to SB4). In all the measurements, the error is the reciprocal of the estimated standard deviation of the pulse duration resulting from the weighted least-square fitting of the cross-correlation curve, where the weight is the standard deviation of the measured cross-correlation curve determined at each data point.

\section{B. High-resolution single-shot solid-state cross-correlation method}

The second method is based on ultrafast optical gating [90]. It involves the use of a solid-state target EUV-optical cross-correlation [91-95]. In such an approach, the FEL pulse is used to excite transient electrons into the conduction band of a dielectric material $\left(\mathrm{Si}_{3} \mathrm{~N}_{4}, \mathrm{SiO}_{2}\right.$, diamond) or semiconductor (GaAs, $\mathrm{Si}$ ). In this experiment, we used $\mathrm{Si}_{3} \mathrm{~N}_{4}$. The wavefront of the FEL is tilted with respect to the target, and the arrival time of each of its portions depends on the spatial positions on the sample. The FEL fluency and the FEL temporal structure are encoded spatially and temporally into the surface of the target.

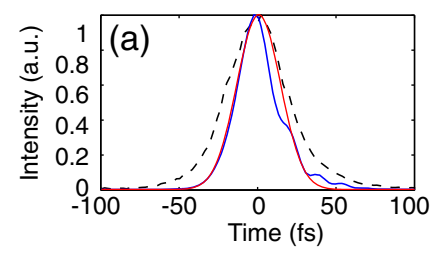

(c)
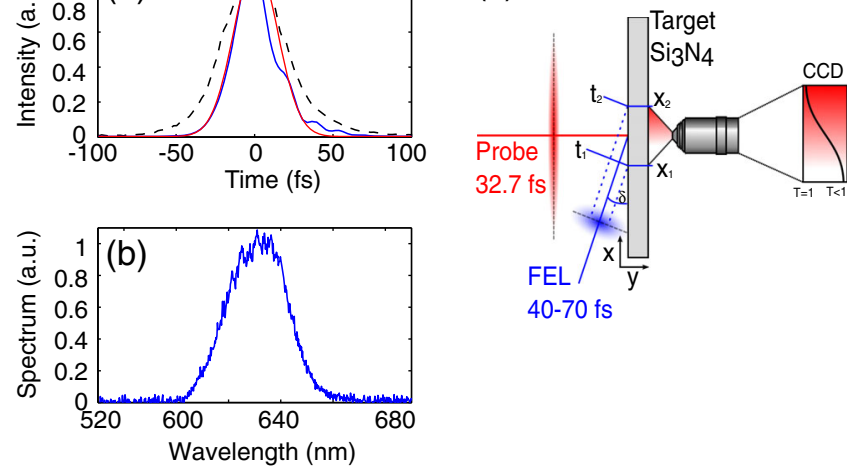

FIG. 9. (a) Autocorrelation signal (dotted black line) of the noncollinear optical parametric amplifier (NOPA) probe, pumped by the FERMI IR laser (784 nm, $100 \mathrm{fs}, 200 \mu \mathrm{J})$. Reconstructed temporal pulse shape from dispersion modeling (blue line) and Gaussian fit of pulse shape (red line). (b) Spectrum of the NOPA signal centered at $630 \mathrm{~nm}$. (c) Schematic of the experimental setup.

The temporal evolution of the FEL excited transient electrons is monitored at the arrival time of a probing optical laser with a wavefront parallel to the target (see Fig. 9). The transmitted light is a cross-correlation between the FEL and the optical pulse $[37,96]$. The transformation between the space $(x)$ and the time $(t)$ coordinate for a given angle $(\delta)$ between the FEL pulse direction and sample surface can be expressed as

$$
t_{2}-t_{1}=\frac{\left(x_{2}-x_{1}\right) \cos (\delta)}{c},
$$

where $c$ is the speed of light. In contrast to other approaches, such as XUV autocorrelation [36,97], spatiotemporal encoding can perform FEL pulse duration measurements on a single-shot basis.

For an accurate determination of the FEL pulse profile, the optical probe pulse must have a known temporal pulse profile $I_{\text {laser }}(t)$. In order to resolve complicated pulse structures, it must be shorter than or comparable to the shortest FEL component. If the probe laser temporal profile is known, the FEL pulse profile can be determined from the cross-correlation function $S(\Delta t)$ given by $[37,90]$

$$
S(\Delta t)=\int_{-\infty}^{\infty} I_{\mathrm{laser}}(\tau) G(\tau-\Delta t) d \tau,
$$

where $G(t)$-the gating function-describes the optical response of the material and contains the information about the temporal evolution of electron density in the conduction band $[98,99]$. It can be evaluated by means of the Fresnel formula for stratified media [90,100], taking into account the dependence of the complex refractive index, $\tilde{\eta}(t, y)=\eta(t, y)+i k(t, y)$, of the layers on the excited electron density $n_{\mathrm{ex}}(t, y)$ through the model of Spitzer and Shkarofsky [101,102]. If the FEL pulse duration is 
much longer than the typical material excitation time, the electron density excited in the conduction band $n_{\mathrm{ex}}(t, y)$ induced by the FEL pulse can be expressed in terms of total FEL absorbed flux, given as [37]

$$
n_{\mathrm{ex}}(t, y)=\frac{\hbar \omega_{\mathrm{FEL}}}{\Delta E_{c}^{*}} \Phi_{\mathrm{ph}}(t) \alpha_{\mathrm{FEL}} e^{-\alpha_{\mathrm{FEL}} y},
$$

where $\hbar \omega_{\mathrm{FEL}}$ is the FEL photon energy, $\Delta E_{c}^{*}$ is the effective energy necessary to promote an electron into the conduction band (taking into account secondary electrons generated by hot electron relaxation [103]), $\alpha_{\mathrm{FEL}}$ is the material absorption coefficient at the FEL photon energy, and $\Phi_{\mathrm{ph}}(t)$ is the time-dependent photon flux irradiating the material. Finally, the total photon flux at time $t$ irradiating the sample can be expressed as the integrated FEL pulse temporal profile $I_{\mathrm{FEL}}(t)$ :

$$
\Phi_{\mathrm{ph}}(t)=\frac{1}{\hbar \omega_{\mathrm{FEL}}} \int_{-t_{0}}^{t} I_{\mathrm{FEL}}\left(t^{\prime}\right) d t^{\prime}
$$

The simulation of the transient electron kinetics in the sample under FEL irradiation was performed by means of the XCASCADE Monte Carlo code [104]. This simulation shows that linear absorption is a valid assumption under our experimental conditions (low fluency). In this particular case, the optical transmission function $G(t)$ for a given $I_{\mathrm{FEL}}(t)$ can be obtained by applying the transfer matrix method to Eqs. (19) and (20) to calculate the complex refractive index [37]. Knowing $G(t)$ and the temporal structure of the probe pulse $I_{\text {laser }}(t)$, a simulated crosscorrelation function $S_{\text {sim }}(\Delta t)$ can be calculated by applying Eq. (18). To finally retrieve the pulse duration from the experimental cross-correlation data $S(\Delta t)$, an optimization routine minimizes the least-square residual error $\left[S_{\mathrm{sim}}(\Delta t)-S(\Delta t)\right]^{2}$ by varying $I_{\mathrm{FEL}}(t)$ and $\Delta t$.

\section{Experimental setup}

Single-shot EUV-optical cross-correlation experiments were carried out at the DiProI end station $[79,80]$. Previous experiments were mostly performed on fused silica [37]. This substrate shows changes of morphology after several shots because the FEL fluency level that is necessary to generate sufficient plasma density is usually above the damage threshold. In this experiment, we used $1-\mu \mathrm{m}$ thick $\mathrm{Si}_{3} \mathrm{~N}_{4}$ membranes, where we could measure below the damage threshold $\left(<0.02 \mathrm{~J} / \mathrm{cm}^{2}\right)$. The $\mathrm{Si}_{3} \mathrm{~N}_{4}$ membrane was mounted on an XYZ stage. The sample was illuminated by FEL radiation arriving with an angle $\delta=20^{\circ}$ with respect to its surface, while the collimated optical probe laser pulse $\left(\lambda_{\text {opt }}=630 \mathrm{~nm}\right)$ was impinged normal to the sample surface with a spot size of 1-mm FWHM (Fig. 9). The FEL beam was focused by the beam-line adaptive optical system [76] to a spot size of about $150 \times 300 \mu \mathrm{m}$, as measured at the sample, with the larger beam dimension on the plane of incidence of the FEL. The transmitted laser pulse was collected by a Mitutoyo $\times 10$ microscope objective and imaged onto a CCD camera triggered with the FEL pulse and placed outside the vacuum vessel to generate an image of the illuminated sample surface. The temporal single-shot field of view of the CCD was $\Delta T=496 \mathrm{fs}$ [see Eq. (17)]. Spatial overlap between the two radiations was checked on a phosphor scintillator screen. A central element of this experiment was an external compact single-stage NOPA, which was installed on the optical breadboard close to the DiProI experimental end station [76]. Since the available pump probe laser at FERMI is limited to $100 \mathrm{fs}$, the NOPA is used to generate shorter optical probe pulses in the range of $30 \mathrm{fs}$ in order to improve the temporal resolution of the experiment. The NOPA was pumped by the second harmonic of the FERMI optical laser at $400 \mathrm{~nm}$ and seeded by white light generated by the fundamental in a 4-mm sapphire crystal. The NOPA was tuned to a $630-\mathrm{nm}$ center wavelength generating $\sim 1 \mu \mathrm{J}$ pulses and compressed in a fused silica prism compressor [105] [Fig. 9(b)]. The temporal structure of the NOPA pulses at the target was determined by a SHG autocorrelation measurement and reconstruction of the pulse shape by precisely modeling the pulse spectral phase using known parameters and optics of the setup [Fig. 9(a)]. At the target, the OPA pulse duration was measured as $(32.7 \pm 1)$ fs $(F W H M)$. Because of losses in the compressor, beam transport, and beam shaping, $300 \mathrm{~nJ}$ of pulse energy reached the target.

\section{Data recording, calibration, and processing}

In the case of XUV photons, the temporal resolution is mainly limited by the optical resolution of the imaging system and by the pulse duration of the optical probe. The secondary electron cascade duration indeed becomes the limiting factor, as analyzed, e.g., in Ref. [98]. The resolution of the imaging system was calibrated with a periodic resolution target (228 cycles per $\mathrm{mm}$ ) placed at the sample plane, with a spatial resolution of $\Delta x=(1.26 \pm 0.97) \mu \mathrm{m}$, leading to a time resolution of $\Delta t=(4 \pm 3)$ fs. The spatiotemporal calibration can be calculated by applying Eq. (17) knowing the crossing angle $\delta$. With the temporal window $\Delta T=496 \mathrm{fs}$ and the number of CCD pixels $\quad N=1626 \mathrm{pxl}, \quad \Delta T / N=(0.305 \pm 0.007) \mathrm{fs} / \mathrm{pxl}$. This value was confirmed by two time-delay scans evaluating the transient transmission edge, resulting in $(0.3061 \pm 0.0032) \mathrm{fs} / \mathrm{pxl}$. The measurement procedure is divided into three steps. First, the transmission of the optical probe laser without a FEL pulse (background) is recorded. Second, the transmission of the optical laser pulse with the FEL pulse at a delay $S_{\text {calibration }}(\Delta t>0, x)$ is recorded, where the probe arrives later than the FEL pulse and the resulting plasma from the FEL is fully developed.

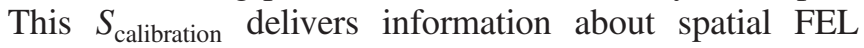
intensity distribution $I_{\mathrm{FEL}}(x)$ and is used to correct the 
signals for spatial features in the FEL profile. Third, the transmission with the FEL pulse at a delay $S_{\text {signal }}(\Delta t=0, x)$ is recorded, where both optical laser and FEL pulses temporally overlap. Initially, both $S_{\text {calibration }}$ and $S_{\text {signal }}$ data need to be background corrected. Then, the spatial-intensitycalibrated cross-correlation $S(\Delta t)$ is produced using the following expression:

$$
S(\Delta t)=1-\frac{1-S_{\text {signal }}}{1-S_{\text {calibration }}}\left(1-\min \left(S_{\text {signal }}\right)\right) .
$$

Figure 10 gives an example of a result of the analysis procedure for one single shot at 26.17-nm center wavelength with a long seeding pulse of $\tau_{\text {seed }}=157.5 \mathrm{fs}$. Note that $S(\Delta t)$ (grey) and the corresponding modeled transmission cross-correlation signal $S(\Delta t)$ (red) are plotted in Fig. 10(a). After an initial decrease of the transmission, it is possible to observe a relaxation of the electron density, which slowly increases the transmission. The plasma relaxation of the electron density (over the range 150-500 fs) has been observed by other groups using fused silica and $\mathrm{Si}_{3} \mathrm{~N}_{4}$ targets [106-108]. However, the appearance of the decay is dependent on the material and on the FEL pulse parameters. In some materials, it is observed only on longer time scales. The "plasma decay" effect is due to the diffusive escape (a)

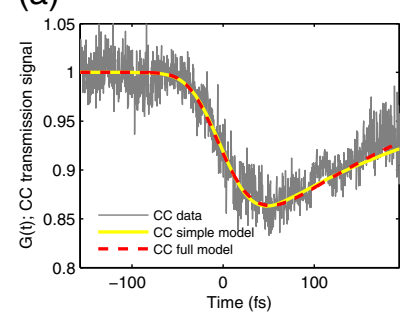

(b)

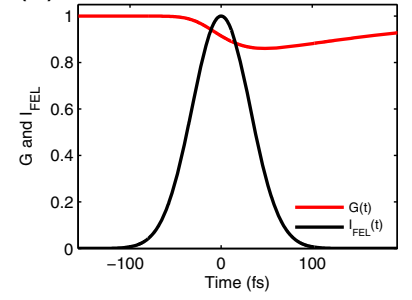

(c)

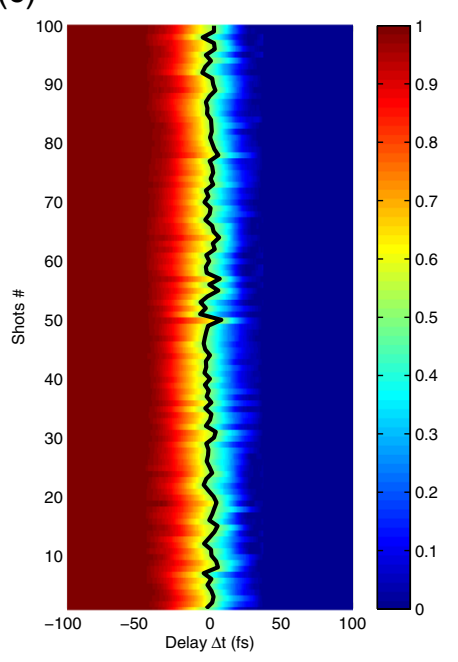

FIG. 10. (a) Single-shot cross-correlation curve from a $\mathrm{Si}_{3} \mathrm{~N}_{4}$ target (grey): $\tau_{\text {seed }}=157.5 \mathrm{fs}$, FEL wavelength $26.17 \mathrm{~nm}$; fits calculated with the cross-correlation simple model (yellow line) and the full-scale cross-correlation function (red line). (b) Solid black line: Retrieved single-shot pulse structure $I_{\mathrm{FEL}}(t)$. Solid red line: FEL gating function. The single-shot pulse duration is $\tau_{\mathrm{FEL}}=$ 74.9 fs. (c) Series of $N=100$ single-shot cross-correlation curves (smoothed) normalized between a transmission (T) of 1 and 0 ; $\tau_{\text {seed }}=112.5 \mathrm{fs}$. The spatial position of the transient edge depends on the relative arrival time between the probe laser and the FEL pulse. Measured average pulse duration $\tau_{\mathrm{FEL}}=(51.2 \pm 2.0) \mathrm{fs}$; average time-of-arrival jitter is $2.2 \mathrm{fs}$ (rms).
[109] of the excited free electrons from the interaction region. Its contribution is closely related to the increasing penetration depth of photons with increasing photon energy, which defines the size of the interaction region. Electrons can escape into the radiation-unexposed part of the silicon nitride layer (1 micron thick), thus rapidly "disappearing" from the interaction region. The photon attenuation length in silicon nitride is $40 \mathrm{~nm}$ at $50-\mathrm{eV}$ photon energy, approximately the photon energy during the FERMI experiments, $100 \mathrm{~nm}$ at $92 \mathrm{eV}$, and between $100 \mathrm{~nm}$ and a few microns for photons ranging between 500 and $2000 \mathrm{eV}$ [110]. In previous experiments at photon energy close to the FERMI energy [111], the return of the transient reflectivity to its initial value was observed on a time scale of $0.5 \mathrm{ps}$. In the experiment [93], after the initial drop, the reflectivity did not recover for at least $3 \mathrm{ps}$. This confirms that the decay effect observed during our measurement at FERMI cannot be due to any inherent recombination process but rather to a diffusion of electrons from the interaction region. The effect of the "plasma decay" influences the gating function $G(t)$ and leads to a systematic shorter pulse duration. We therefore addressed this problem by introducing an exponential relaxation term in the simulation. From the analysis of the transmission signal by means of this modified model function, we determined a decay factor $\tau_{\text {decay }}=(239.2 \pm 15.6)$ fs. The decay factor is modeled as a time-dependent electron density parameter included [Eq. (18)]. Calculating the full scale modeling [37] of the cross-correlation functions is time consuming. Therefore, in the case of the long-scan series, we applied a simplified function (simple model) for fitting the experimental crosscorrelation curves. Since the experiment was performed in a linear absorption regime and at optimized FEL conditions generating single Gaussian pulses, the simple model could be reduced to a two-parameter $\left(\Delta t, \tau_{\mathrm{FEL}}\right)$ Gauss-fit model. The simple model was cross-calibrated with a reduced number of full-scale model results with different $\tau_{\mathrm{FEL}}$. The FWHM pulse duration parameter $\tau_{\mathrm{FEL}}$ resulting from the full-scale model differs by a constant factor from the result of the simple model. This deviation is taken into account by correcting the simple model with a calibration factor, which accounts for the small discrepancy between the two models. A comparison between the simple model (yellow line) and the full scale model (red line) is shown in Fig. 10(a). In Fig. 10(b), the reconstructed pulse and the corresponding retrieved gating function are shown. In addition to the pulse duration measurement, the relative time of arrival for each shot has been extracted. The temporal delay between the FEL pulse and probe laser pulse was determined by the spatial position of the turning point of the cross-correlation curve. The influence of the FEL pointing jitter can be neglected because the deviation of the time of arrival is estimated to be smaller than $1 \mathrm{fs}$. As an example, a series of 100 shots with seed pulse duration $\left(\tau_{\text {seed }}=112 \mathrm{fs} \pm 2.5 \mathrm{fs}\right)$ has provided a FEL pulse duration 
$\tau_{\mathrm{FEL}}=(51 \pm 2)$ fs at $26.2 \mathrm{~nm}$, with a time-of-arrival jitter of 2.2 fs rms [Fig. 10(c)].

\section{EXPERIMENTAL RESULTS}

Measurements with the two methods were carried out in different machine conditions and at different FEL output wavelengths. Different seed pulse durations and different seed pulse generation schemes (OPA or THG) were used for the measurements. As pointed out in Sec. II, the final pulse length is expected to depend on the seed pulse duration and on the FEL harmonic conversion order. The up-shift to a harmonic of order $n$ causes a reduction of the optical pulse duration scaling as $1 / \sqrt{n}$ when the FEL is operated far from saturation both in the modulator and/or dispersion section and in the final amplifier. When the output pulse is optimized (maximum bunching at minimum energy modulation, $\left.\chi_{n} \sim \chi_{n}^{\max }\right)$, the pulse length is expected to scale as about $7 /\left(6 n^{1 / 3}\right)$. In deeply saturated conditions, induced by the modulator-dispersion or by the dynamics in the final amplifier, the pulse length grows with the formation of pulse structures. The level of saturation can be controlled by varying the intensity of the seed laser or the dispersion after the modulator. Both of these parameters control the amplitude of the bunching factor at the entrance of the final amplifier. At the same time, the level of saturation can be monitored by measuring the extracted energy per pulse and by observing the spectral signatures of saturation effects, such as spectral broadening and the appearance of substructures in the spectral profile [49]. In Table I, we report a summary of measurements obtained in conditions of almost-single-mode spectra with a pulse energy substantially lower than the maximum available $(50 \%-60 \%$, mode $=\mathbf{n})$ and measurements in partially saturated conditions (mode $=\mathbf{s}$ ). These results are therefore expected to be free of "deep" saturation effects. The measurements with method A require the acquisition of data points for different delay times between the FEL and IR pulses. With method B, each measurement is the result

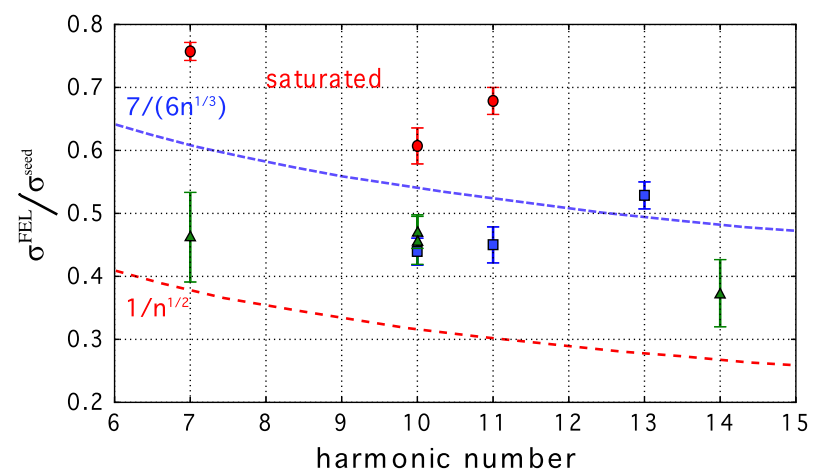

FIG. 11. Summary of measurements. Blue squares show method A; green triangles show method B. These measurements are associated with data in Table I corresponding to the normal "optimized" configuration (mode $=\mathbf{n}$ ). Red dashed line: The function $1 / \sqrt{n}$. Blue dotted line: Optimized peak bunching factor, scaling as about $7 /\left(6 n^{1 / 3}\right)$. The red points, obtained with an excess of seed power or dispersion, causing an evident broadening in the spectrum (mode $=\mathrm{s}$ in Table I), are instead characterized by a systematic larger duration.

of an average over 100 single-shot acquisitions, and the method also provides an estimate of the arrival-time jitter $\tau_{a}$.

In Fig. 11, we plot the ratio between the measured FEL pulse length and the seed pulse length, as a function of the harmonic order $n$ for the data in Table I. The measured pulse durations in blue and green $($ mode $=n)$ are $15 \%$ to $22 \%$ lower than the duration estimated in the expected optimized conditions, Eq. (13) $\left[\tau_{\mathrm{FEL}} \sim 7 \tau_{\text {seed }} /\left(6 n^{1 / 3}\right)\right]$. The data point corresponding to harmonic 13 , which shows a slightly larger duration than the $7 /\left(6 n^{1 / 3}\right)$ trend, was measured on FEL-2.1. The anomaly could be associated with a tuning of the FEL, with an increased seed intensity compensating for the lower gain in the amplifier of FEL-2.1 with respect to FEL-1. These measurements suggest that the FEL optimization based on the criterion of a narrow,

TABLE I. FEL pulse duration data, measured with method $(M)$ A or B, with the harmonic $(n)$ corresponding to the wavelength $\left(\lambda_{\mathrm{FEL}}\right)$. Seeds at wavelengths $\left(\lambda_{\text {seed }}\right) 261.1 \mathrm{~nm}$ and $261.7 \mathrm{~nm}$ were generated in THG, while in the other cases, the OPA was used. Arrival-time jitter $\left(\tau_{a}\right)$ is for method B only. The column mode indicates the saturation conditions: $\mathbf{n}=$ normal, $\mathbf{s}=$ saturated.

\begin{tabular}{|c|c|c|c|c|c|c|c|}
\hline$M$ & $n$ & $\lambda_{\text {seed }}(\mathrm{nm})$ & $\lambda_{\mathrm{FEL}}(\mathrm{nm})$ & $\tau_{\text {seed }}(\mathrm{fs})$ & $\tau_{\mathrm{FEL}}(\mathrm{fs})$ & $\tau_{a}(\mathrm{fs})$ & Mode \\
\hline A & 10 & 257.8 & 25.78 & 140 & $61.5 \pm 3$ & $\ldots$ & n \\
\hline A & 11 & 261.1 & 23.74 & 140 & $63 \pm 4$ & $\ldots$ & n \\
\hline A & 13 & 261.1 & 20.08 & 140 & $74 \pm 3$ & $\ldots$ & n \\
\hline B & 7 & 261.7 & 37.38 & 112.5 & $52 \pm 8$ & $\ldots$ & n \\
\hline B & 10 & 261.7 & 26.17 & 112.5 & $53 \pm 3$ & $3 \pm 1$ & n \\
\hline B & 10 & 261.7 & 26.17 & 157.5 & $72 \pm 6$ & $8 \pm 2$ & n \\
\hline B & 14 & 261.7 & 18.69 & 112.5 & $42 \pm 6$ & $\cdots$ & n \\
\hline $\mathrm{A}$ & 7 & 261.1 & 37.30 & $140 \pm 2.5$ & $106 \pm 2$ & $\ldots$ & $\mathbf{s}$ \\
\hline A & 10 & 257.8 & 25.78 & $140 \pm 2.5$ & $85 \pm 4$ & $\ldots$ & $\mathbf{s}$ \\
\hline A & 11 & 261.1 & 23.74 & $140 \pm 2.5$ & $95 \pm 3$ & $\cdots$ & $\mathbf{s}$ \\
\hline
\end{tabular}


Gaussian-like spectrum, without side peaks, leads to a working point with a corresponding $\chi_{n} / \chi_{n}^{\max }$ factor [Eq. (11)] of about 0.8-1.0. The other data points (red, mode $=s$ in the table) refer to measurements where the FEL was tuned, on purpose, with an increased seed power and/or dispersion or, in other words, with $\chi_{n} / \chi_{n}^{\max }>1$ [Eq. (11)] (see Table I). These measurements show a $10 \%-20 \%$ longer pulse duration.

From independent spectral measurements of the FEL pulses, a Fourier transform (FT)-limited pulse duration can be calculated according to the relation

$$
\tau_{\mathrm{FTL}} \simeq 0.44 \lambda^{2} /(c \Delta \lambda) .
$$

The $\tau_{\text {FTL }}$ pulse values are systematically shorter than the measured ones by $30 \%$ for the cases corresponding to the THG seed and by up to $60 \%$ for the OPA seeding scheme. This difference is due to the residual chirp in the seed laser pulse spectral width, leading to FEL pulses that are not FT limited in the conditions of the measurements. The OPA seeding mode shows a larger frequency chirp than the THG setup.

In the OPA seeding mode, the linear component of the frequency chirp was large enough to correlate the temporal pulse profile with the spectral pulse profile [69]. In Fig. 12(a), the cross-correlation curve measured (with method A) at harmonic $n=6(42.97 \mathrm{~nm})$ in deeply saturated conditions is compared to the pulse spectrum. The spectral profile shows a clear correspondence between time and wavelength with a spectrotemporal splitting of the pulse. The spectral intensity profile shows a double peak, with the minor component of the spectrum containing about $15 \%$ of the total intensity. The FEL pulse head is the rising edge on the right side of Fig. 12(a). This component corresponds to the most intense component in the spectral profile, which lies at longer wavelength [Fig. 12(b)] and is consistent with a positive chirp of the seed pulse. By means of peak fitting, the
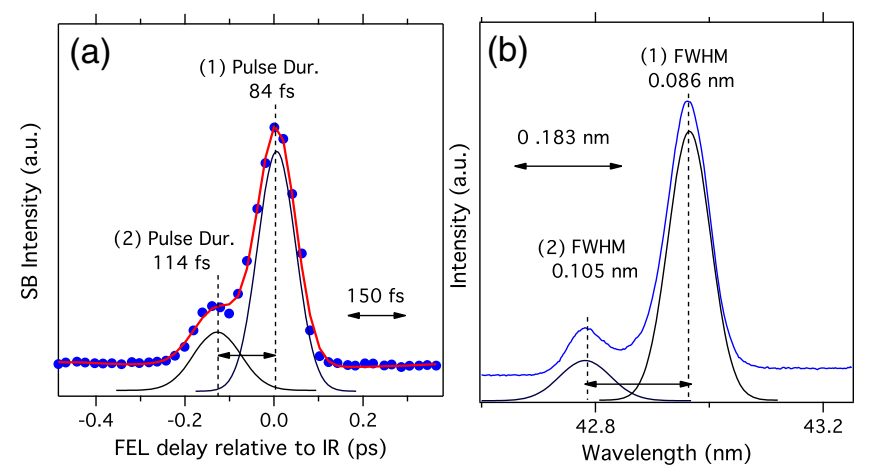

FIG. 12. (a) Cross-correlation curve at a wavelength $\lambda_{\text {FEL }}=$ $42.96 \mathrm{~nm}$ (6th harmonics) for the source FEL-1 measured with method A. (b) Wavelength dispersion of the pulse (average over 200 shots) as measured with the beam-line spectrometer PRESTO. Temporal and wavelength profiles were fitted with two Gaussian peaks (solid black lines). wavelength span is correlated with the time span. From the plot, we may estimate a linear frequency chirp rate $\alpha \sim$ $6.2 \times 10^{26} \mathrm{rad} / \mathrm{s}^{2}$ defined as $\omega(t)=[d /(d t)] \varphi(t)=\omega_{0}+2 \alpha t$, where $\omega(t)$ describes the linear frequency shift along the pulse centered at $\omega_{0}$ (this value of $\alpha$ corresponds to a group delay dispersion of about $800 \mathrm{fs}^{2}$ ).

As anticipated, method B allows a correlation of the pulse length to the spectral width on a shot-to-shot basis. The results shown in Fig. 13 were taken with the THG seeding mode, with a seed pulse duration $\tau_{\text {seed }}=$ $(112 \pm 2.5) \mathrm{fs}$ and FEL set at harmonic $n=10$ $(26.17 \mathrm{~nm})$. The plot corresponds to a sample of 200 shots, where for each FEL pulse, a corresponding spectrum and pulse duration were acquired. The black dashed line shows the bandwidth vs. the pulse duration for FT-limited Gaussian pulses. The time-bandwidth product of the FEL pulses corresponding to the measured data points (blue dots) is about $30 \%$ larger than the FT limit. This is compatible with the assumption of a residual linear frequency chirp rate $\alpha \sim 4 \times 10^{26} \mathrm{rad} / \mathrm{s}^{2}$ (red solid line), which may be attributed to the residual frequency chirp of the seed laser pulse. In this set of measurements, the seed pulse bandwidth was indeed $1.15 \mathrm{~nm}$ FWHM, indicating a time-bandwidth product $1.28 \times$ FT limit. For a thorough discussion about the effect of the chirp in a seeded FEL and about the generation of FT-limited pulses, see also Refs. [43,49].

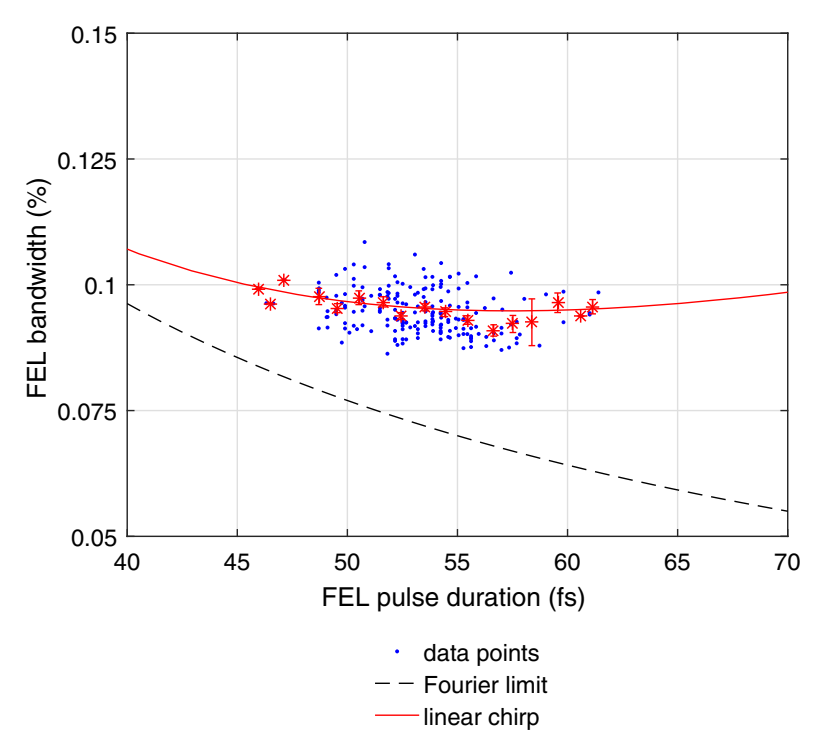

FIG. 13. Measured FEL pulse duration and bandwidth of 200 consecutive shots at fixed machine conditions. Blue dots are the measured data, the dashed black line shows the theoretical curve for FEL pulses with a perfect FT-limited Gaussian profile, and the solid red line is the bandwidth as a function of the duration of an ideal Gaussian pulse with chirp $\alpha \sim 4 \times 10^{26} \mathrm{rad} / \mathrm{s}^{2}$ (corresponding to a group delay dispersion of about $540 \mathrm{fs}^{2}$ ). All FEL pulse durations are measured with the single-shot cross-correlation. The red stars represent the grouped average of the data points. 

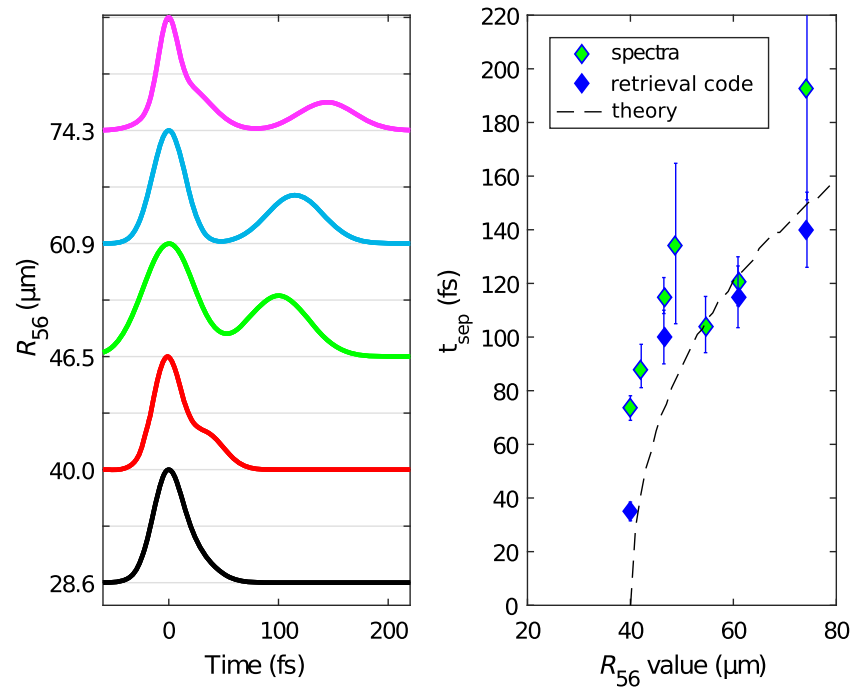

FIG. 14. Pulse temporal profile as a function of the $R_{56}$ parameter. Left panel: Single-shot cross-correlation retrieved FEL temporal pulse profile. Right panel: Temporal separation of the split pulses at $R_{56}>40 \mu \mathrm{m}$ (blue diamonds); separation calculated from Eq. (9) under the assumption that $\chi_{n}=\chi_{n}^{\max }$ is obtained at $R_{56}=40 \mu \mathrm{m}$ (black dashed line); pulse separation estimated from the spectral traces in Fig. 15 (green diamonds).

In similar conditions, i.e., $\tau_{\text {seed }}=(112 \pm 2.5)$ fs at $n=$ 10 , the dispersion parameter $R_{56}$ was increased from $26.2 \mu \mathrm{m}$ up to $75 \mu \mathrm{m}$. This corresponds to an increase of about 2.9 of the $\chi_{n} / \chi_{n}^{\max }$ factor [Eq. (11)], driving a previously optimized condition into a deeply saturated condition. This setting is expected to induce the formation of multiple structures in the FEL pulse (see Fig. 2). Figure 14(a) shows a measurement of the cross-correlation data. Starting at $R_{56}=46.5 \mu \mathrm{m}$, the pulse profile shows a well-separated secondary peak. At larger values of $R_{56}$, the pulse clearly splits into two substructures whose separation grows with the dispersion $R_{56}$. In Fig. 14(b), the pulse separation resulting from the profiles in Fig. 14(a) is compared to the separation calculated from Eq. (9), under the assumption that the condition $\chi_{n}=$ $\chi_{n}^{\max }$ is obtained at $R_{56}=40 \mu \mathrm{m}$ [see the red solid line in Fig. 14(b)]. We observe two main pulses with a separation in reasonable agreement with the theory. An indirect way of retrieving the separation between the two peaks is via the spectral data. The pulse spectra, measured simultaneously and independently via the spectrometer (see Fig. 15), show the presence of intensity modulations, suggesting a structure in the temporal distribution [49]. We made the rough assumption that the splitting leads to the formation of two identical Gaussian pulses separated in time by an interval $\delta t$, with a common frequency chirp. The chirp is derived from the analysis of Fig. 13. We may therefore calculate the separation $\delta t$ from the period of the spectral modulation. For the reconstruction of the temporal separation $\delta t$, we assumed the pulse was composed of two identical Gaussian pulses of rms duration $\sigma_{t}$ with a common linear frequency chirp $\alpha$,

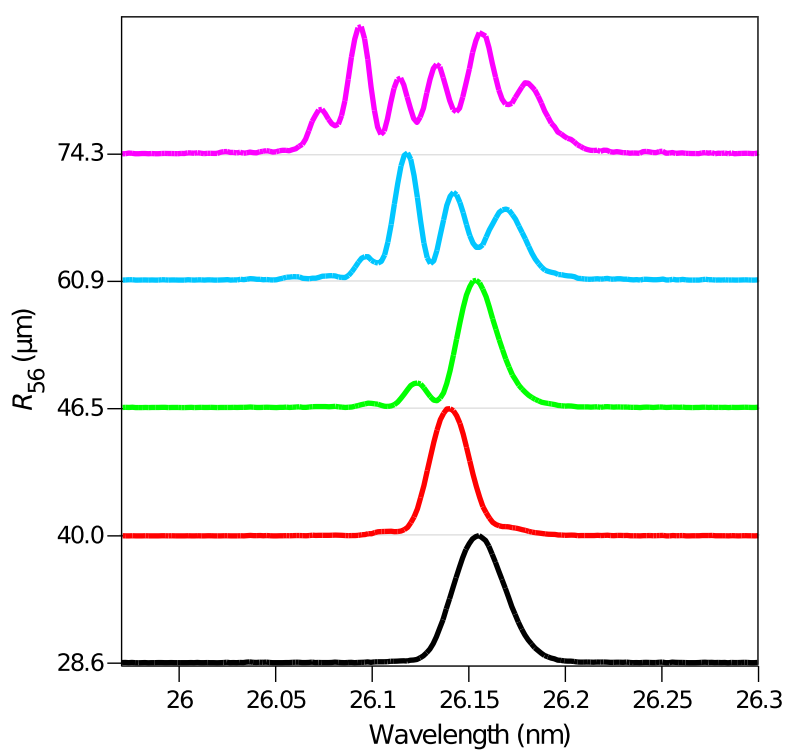

FIG. 15. Spectrum of the radiation at the corresponding values of dispersion as shown in Fig. 14. Each image is the result of an average over five single-shot acquisitions; the spectral features are relatively stable in time.

$E(t)=E_{0}\left\{e^{-(t+\delta t / 2)^{2} / 4 \sigma_{t}^{2}}+e^{-(t-\delta t / 2)^{2} / 4 \sigma_{t}^{2}}\right\} \exp [i(\omega+2 \alpha t) t]$. The period of the spectral modulation depends on the chirp and on the temporal separation $T_{\omega}=[(2 \pi) / \delta t]\left[1+\left(4 \alpha \sigma_{t}^{2}\right)^{2}\right]$. The value of $\sigma_{t}$ was retrieved from the leading pulse profile in Fig. 14(a). The temporal separation reconstructed from the spectral data is shown in Fig. 14(b) and is comparable to the value directly measured with method $B$.

\section{CONCLUDING REMARKS}

We have studied the pulse temporal shape and expected behavior of the pulse duration in a seeded FEL operating in the HGHG configuration. We have measured this parameter with two reliable methods: two-color photoemission cross-correlation (method A, Sec. III A) and highresolution single-shot solid-state cross-correlation (method B, Sec. III B). The results obtained with the two methods were mutually consistent. Both methods can determine "online" the temporal pulse profile of a FEL, as would be the case for XUV autocorrelation methods. In particular, method B was demonstrated in a single-shot mode. Pulse characteristics, such as the pulse splitting at saturation, were uniquely determined: See Fig. 12 for method A and Fig. 14 for method B.

In the framework of the optimized operating conditions of the seeded FEL FERMI, the theoretical predictions for the pulse evolution have been compared to the results. The measurements were carried out at different wavelengths and for different machine settings and seed laser pulse durations. We may distinguish between a short pulse mode of operation - characterized by a single-mode narrow-band spectrum and reduced energy per pulse of the order of $50 \%$ 
of the maximum attainable energy - and a high energy mode. In the first case, the FEL pulse duration at the harmonic $n$ was measured to be typically shorter than $\tau_{\mathrm{FEL}} \sim\left(\frac{7}{6}\right) \tau_{\text {seed }} / n^{1 / 3}$, as predicted by theory and regardless of the THG or OPA seeding mode of operation. For those settings, such that a single Gaussian mode can be observed in the spectrum, pulse lengths well below the 100 -fs regime could be systematically measured independently of the specific seed setup. In the second case, the higher energy per pulse comes with a pulse duration that can even be 2-3 times larger (see Fig. 14) and with the pulse showing a more complicated temporal structure, also reflecting on the spectral properties of the light. As expected, the correlation with the spectral features depends on the frequency chirp of the pulse and, indirectly, on the spectral chirp of the seed laser. The FEL linear frequency chirp was estimated at harmonic $n=6$, in deeply saturated conditions, seeded by the larger chirp of the OPA laser setup, and at $n=10$, with the THG setup. In addition, at $n=10$, an $R_{56}$ scan was carried out. At large values of $R_{56}$, two main pulses with a separation in agreement with theoretical prediction were observed. The above experimental techniques have therefore shown the capability to provide temporal measurements, even in the presence of complex pulse structures (with multiple peaks both in the temporal and spectral domains), whenever they were induced by specific tuning of the machine parameters. Thus, transient transmission cross-correlation may provide a tool to link the spectral shape to the pulse temporal structure on a single-shot basis.

Knowledge of the temporal pulse profile is of paramount importance for FEL studies aimed at deepening science of time-resolved phenomena. The methods used in the setup tested at FERMI can be implemented as online diagnostics for short-wavelength FELs, regardless of the seeded or SASE mode of operation. Concerning the unique seeded FEL source FERMI, new facilities implementing the external seed to improve longitudinal coherence were proposed in the past few years and are now coming into operation, such as the seeded FLASH upgrade at DESY [112] and the Dalian Coherent Light Source (DCLS) [113]. Exact knowledge of the interplay of the machine parameters governing the pulse properties in the seeded mode is therefore an important aspect for both the scientists proposing experiments requiring the unique properties of a seeded FEL and for the FEL tuning process itself, with the determination of the pulse intensity and the possibility of an online, single-shot, temporal pulse characterization to perform, e.g., slow-loop feedback with long-term-stability FEL pulses.

\section{ACKNOWLEDGMENTS}

The authors wish to thank the FERMI team for the support and cooperation in running the light source. This work has been financially supported by the Swedish
Research Council (VR) and the Knut and Alice Wallenberg Foundation, Sweden.

[1] L. Young, E. P. Kanter, B. Krässig, Y. Li, A. M. March, S. T. Pratt, R. Santra, S. H. Southworth, N. Rohringer, L. F. DiMauro et al., Femtosecond Electronic Response of Atoms to Ultra-Intense X-Rays, Nature (London) 466 , 56 (2010).

[2] M. Meyer, D. Cubaynes, V. Richardson, J. T. Costello, P. Radcliffe, W. B. Li, S. Düsterer, S. Fritzsche, A. Mihelic, K. G. Papamihail, and P. Lambropoulos, Two-Photon Excitation and Relaxation of the $3 d-4 d$ Resonance in Atomic Kr, Phys. Rev. Lett. 104, 213001 (2010).

[3] H. N. Chapman, P. Fromme, A. Barty, T. A. White, R. A. Kirian, A. Aquila, M. S. Hunter, J. Schulz, D. P. DePonte, U. Weierstall et al., Femtosecond X-Ray Protein Nanocrystallography, Nature 470, 73 (2011)., Femtosecond X-Ray Protein Nanocrystallography, Nature (London) 470, 73 (2011).

[4] N. Berrah, L. Fang, B. Murphy, T. Osipov, K. Ueda, E. Kukk, R. Feifel, P. van der Meulen, P. Salen, H. T. Schmidt et al., Double-Core-Hole Spectroscopy for Chemical Analysis with an Intense X-Ray Femtosecond Laser, Proc. Natl. Acad. Sci. U.S.A. 108, 16912 (2011).

[5] M. Nakano, F. Penent, M. Tashiro, T. P. Grozdanov, M. Žitnik, S. Carniato, P. Selles, L. Andric, P. Lablanquie, J. Palaudoux et al., Single Photon $K^{-2}$ and $K^{-1} K^{-1}$ Double Core Ionization in $\mathbf{C}_{2} \mathbf{H}_{2 n}(n=1-3), C O$, and $\mathbf{N}_{2}$ as a Potential New Tool for Chemical Analysis, Phys. Rev. Lett. 110, 163001 (2013).

[6] B. K. McFarland, J. P. Farrell, S. Miyabe, F. Tarantelli, A. Aguilar, N. Berrah, C. Bostedt, J. D. Bozek, P. H. Bucksbaum, J. C. Castagna et al., Ultrafast X-Ray Auger Probing of Photoexcited Molecular Dynamics, Nat. Commun. 54235 (2014).

[7] C. E. Liekhus-Schmaltz, I. Tenney, T. Osipov, A. SanchezGonzalez, N. Berrah, R. Boll, C. Bomme, C. Bostedt, J. D. Bozek, S. Carron et al., Ultrafast Isomerization Initiated by X-Ray Core Ionization, Nat. Commun. 6, 8199 (2015).

[8] E. Allaria, R. Appio, L. Badano, W. A. Barletta, S. Bassanese, S. G. Biedron, A. Borga, E. Busetto, D. Castronovo, P. Cinquegrana et al., Highly Coherent and Stable Pulses from the FERMI Seeded Free-Electron Laser in the Extreme Ultraviolet, Nat. Photonics 6, 699 (2012).

[9] E. Allaria, D. Castronovo, P. Cinquegrana, P. Craievich, M. Dal Forno, M. B. Danailov, G. D'Auria, A. Demidovich, G. De Ninno, S. Di Mitri et al., Two-Stage Seeded SoftX-Ray Free-Electron Laser, Nat. Photonics 7, 913 (2013).

[10] E. Allaria, F. Bencivenga, R. Borghes, F. Capotondi, D. Castronovo, P. Charalambous, P. Cinquegrana, M. B. Danailov, G. De Ninno, A. Demidovich et al., TwoColour Pump-Probe Experiments with a Twin-Pulse-Seed Extreme Ultraviolet Free-Electron Laser, Nat. Commun. 4, 2476 (2013).

[11] T. Mazza, M. Ilchen, A. J. Rafipoor, C. Callegari, P. Finetti, O. Plekan, K. C. Prince, R. Richter, M. B. Danailov, A. Demidovich et al., Determining the Polarization State of an Extreme Ultraviolet Free-Electron Laser Beam 
Using Atomic Circular Dichroism, Nat. Commun. 5, 3648 (2014).

[12] Y. Ovcharenko, V. Lyamayev, R. Katzy, M. Devetta, A. LaForge, P. O'Keeffe, O. Plekan, P. Finetti, M. Di Fraia, M. Mudrich et al., Novel Collective Autoionization Process Observed in Electron Spectra of He Clusters, Phys. Rev. Lett. 112, 073401 (2014).

[13] M. Žitnik, A. Mihelič, K. Bučar, M. Kavčič, , J.-E. Rubensson, M. Svanquist, J. Söderström, R. Feifel, C. Såthe, Y. Ovcharenko et al., High Resolution Multiphoton Spectroscopy by a Tunable Free-Electron-Laser Light, Phys. Rev. Lett. 113, 193201 (2014).

[14] C. von Korff Schmising, B. Pfau, M. Schneider, C. M. Günther, M. Giovannella, J. Perron, B. Vodungbo, L. Müller, F. Capotondi, E. Pedersoli, N. Mahne, J. Lüning, and S. Eisebitt, Imaging Ultrafast Demagnetization Dynamics after a Spatially Localized Optical Excitation, Phys. Rev. Lett. 112, 217203 (2014).

[15] F. Bencivenga, R. Cucini, F. Capotondi, A. Battistoni, R. Mincigrucci, E. Giangrisostomi, A. Gessini, M. Manfredda, I. P. Nikolov, E. Pedersoli et al., Four-Wave Mixing Experiments with Extreme Ultraviolet Transient Gratings, Nature (London) 520, 205 (2015).

[16] K. C. Prince, E. Allaria, C. Callegari, R. Cucini, G. De Ninno, S. Di Mitri, B. Diviacco, E. Ferrari, P. Finetti, D. Gauthier et al., Coherent Control with a Short-Wavelength FreeElectron Laser, Nat. Photonics 10, 176 (2016).

[17] N. Berrah, J. Bozek, J. T. Costello, S. Düsterer, L. Fang, J. Feldhaus, H. Fukuzawa, M. Hoener, Y. H. Jiang, P. Johnsson et al., Non-linear Processes in the Interaction of Atoms and Molecules with Intense EUV and X-Ray Fields from SASE Free Electron Lasers (FELs), J. Mod. Opt. 57, 1015 (2010).

[18] B. Nagler, U. Zastrau, R. R. Fäustlin, S. M. Vinko, T. Whitcher, A. J. Nelson, R. Sobierajski, J. Krzywinski, J. Chalupsky, E. Abreu et al., Turning Solid Aluminium Transparent by Intense Soft X-Ray Photoionization, Nat. Phys. 5, 693 (2009).

[19] R. Neutze, R. Wouts, D. van der Spoel, E. Weckert, and J. Hajdu, Potential for Biomolecular Imaging with Femtosecond X-Ray Pulses, Nature (London) 406, 752 (2000).

[20] C. Caleman, G. Huldt, F. R. N. C. Maia, C. Ortiz, F. G. Parak, J. Hajdu, D. van der Spoel, H. N. Chapman, and N. Timneanu, On the Feasibility of Nanocrystal Imaging Using Intense and Ultrashort X-Ray Pulses, ACS Nano 5, 139 (2011).

[21] K. H. Bennemann, Ultrafast Dynamics in Solids, J. Phys. Condens. Matter 16, R995 (2004).

[22] J. Hohlfeld, S.-S. Wellershoff, J. Güdde, U. Conrad, V. Jähnke, and E. Matthias, Electron and Lattice Dynamics Following Optical Excitation of Metals, Chem. Phys. 251, 237 (2000).

[23] K. H. Bennemann, Photoinduced Phase Transitions, J. Phys. Condens. Matter 23, 073202 (2011).

[24] N. Medvedev, U. Zastrau, E. Förster, D. O. Gericke, and B. Rethfeld, Short-Time Electron Dynamics in Aluminum Excited by Femtosecond Extreme Ultraviolet Radiation, Phys. Rev. Lett. 107, 165003 (2011).

[25] N. Medvedev and B. Rethfeld, Transient Dynamics of the Electronic Subsystem of Semiconductors Irradiated with an Ultrashort Vacuum Ultraviolet Laser Pulse, New J. Phys. 12, 073037 (2010).

[26] L. Müller, C. Gutt, B. Pfau, S. Schaffert, J. Geilhufe, F. Büttner, J. Mohanty, S. Flewett, R. Treusch, S. Düsterer et al., Breakdown of the X-Ray Resonant Magnetic Scattering Signal During Intense Pulses of Extreme Ultraviolet Free-Electron-Laser Radiation, Phys. Rev. Lett. 110, 234801 (2013).

[27] T. Wang, D. Zhu, B. Wu, C. Graves, S. Schaffert, T. Rander, L. Müller, B. Vodungbo, C. Baumier, D. P. Bernstein et al., Femtosecond Single-Shot Imaging of Nanoscale Ferromagnetic Order in Co/Pd Multilayers Using Resonant X-Ray Holography, Phys. Rev. Lett. 108, 267403 (2012).

[28] J. Gaudin, N. Medvedev, J. Chalupský, T. Burian, S. Dastjani-Farahani, V. Hájková, M. Harmand, H. O. Jeschke, L. Juha, M. Jurek et al., Photon Energy Dependence of Graphitization Threshold for Diamond Irradiated with an Intense XUV FEL Pulse, Phys. Rev. B 88, 060101 (2013).

[29] B. F. Murphy, T. Osipov, Z. Jurek, L. Fang, S. K. Son, M. Mucke, J. H. D. Eland, V. Zhaunerchyk, R. Feifel, L. Avaldi et al., Femtosecond X-Ray-Induced Explosion of $\mathrm{C}_{60}$ at Extreme Intensity, Nat. Commun. 5, 4281 (2014).

[30] L. Fang, T. Osipov, B. Murphy, F. Tarantelli, E. Kukk, J. P. Cryan, M. Glownia, P. H. Bucksbaum, R. N. Coffee, M. Chen, C. Buth, and N. Berrah, Multiphoton Ionization as a Clock to Reveal Molecular Dynamics with Intense Short X-Ray Free Electron Laser Pulses, Phys. Rev. Lett. 109, 263001 (2012).

[31] L. J. Frasinski, V. Zhaunerchyk, M. Mucke, R. J. Squibb, M. Siano, J. H. D. Eland, P. Linusson, P. v. d. Meulen, P. Salén, R. D. Thomas et al., Dynamics of Hollow Atom Formation in Intense X-Ray Pulses Probed by Partial Covariance Mapping, Phys. Rev. Lett. 111, 073002 (2013).

[32] C. Behrens, N. Gerasimova, Ch. Gerth, B. Schmidt, E. A. Schneidmiller, S. Serkez, S. Wesch, and M. V. Yurkov, Constraints on Photon Pulse Duration from Longitudinal Electron Beam Diagnostics at a Soft X-Ray Free-Electron Laser, Phys. Rev. ST Accel. Beams 15, 030707 (2012).

[33] C. Behrens, F. J. Decker, Y. Ding, V. A. Dolgashev, J. Frisch, Z. Huang, P. Krejcik, H. Loos, A. Lutman, T. J. Maxwell, J. Turner, J. Wang, M. H. Wang, J. Welch, and J. $\mathrm{Wu}$, Few-Femtosecond Time-Resolved Measurements of X-Ray Free-Electron Lasers, Nat. Commun. 5, 3762 (2014).

[34] A. A. Lutman, Y. Ding, Y. Feng, Z. Huang, M. Messerschmidt, J. Wu, and J. Krzywinski, Femtosecond X-Ray Free Electron Laser Pulse Duration Measurement from Spectral Correlation Function, Phys. Rev. ST Accel. Beams 15, 030705 (2012).

[35] Y. Inubushi, K. Tono, T. Togashi, T. Sato, T. Hatsui, T. Kameshima, K. Togawa, T. Hara, T. Tanaka, H. Tanaka, T. Ishikawa, and M. Yabashi, Determination of the Pulse Duration of an X-Ray Free Electron Laser Using Highly Resolved Single-Shot Spectra, Phys. Rev. Lett. 109, 144801 (2012).

[36] R. Mitzner, A. A. Sorokin, B. Siemer, S. Roling, M. Rutkowski, H. Zacharias, M. Neeb, T. Noll, F. Siewert, 
W. Eberhardt, M. Richter, P. Juranic, K. Tiedtke, and J. Feldhaus, Direct Autocorrelation of Soft-X-Ray FreeElectron-Laser Pulses by Time-Resolved Two-Photon Double Ionization of He, Phys. Rev. A 80, 025402 (2009).

[37] R. Riedel, A. Al-Shemmary, M. Gensch, T. Golz, M. Harmand, N. Medvedev, M. J. Prandolini, K. SokolowskiTinten, S. Toleikis, U. Wegner et al., Single-Shot Pulse Duration Monitor for Extreme Ultraviolet and X-Ray Free-Electron Lasers, Nat. Commun. 4, 1731 (2013).

[38] U. Frühling, M. Wieland, M. Gensch, T. Gebert, B. Schütte, M. Krikunova, R. Kalms, F. Budzyn, O. Grimm, J. Rossbach et al., Single-Shot Terahertz-Field-Driven X-Ray Streak Camera, Nat. Photonics 3, 523 (2009).

[39] I. Grguraš, A. R. Maier, C. Behrens, T. Mazza, T. J. Kelly, P. Radcliffe, S. Düsterer, A. K. Kazansky, N. M. Kabachnik, Th. Tschentscher et al., Ultrafast X-Ray Pulse Characterization at Free-Electron Lasers, Nat. Photonics 6, 852 (2012).

[40] F. Tavella, N. Stojanovic, G. Geloni, and M. Gensch, FewFemtosecond Timing at Fourth-Generation X-Ray Light Sources, Nat. Photonics 5, 162 (2011).

[41] S. Düsterer, P. Radcliffe, C. Bostedt, J. Bozek, A. L Cavalieri, R. Coffee, J. T. Costello, D. Cubaynes, L. F. DiMauro, Y. Ding et al., Femtosecond X-Ray Pulse Length Characterization at the Linac Coherent Light Source FreeElectron Laser, New J. Phys. 13, 093024 (2011).

[42] W. Helml, A. R. Maier, W. Schweinberger, I. Grguraš, P. Radcliffe, G. Doumy, C. Roedig, J. Gagnon, M. Messerschmidt, S. Schorb, C. Bostedt, F. Grüner, L. F. DiMauro, D. Cubaynes, J. D. Bozek, Th. Tschentscher, J. T. Costello, M. Meyer, R. Coffee, S. Düsterer, A. L. Cavalieri, and R. Kienberger, Measuring the Temporal Structure of Few-Femtosecond Free-Electron Laser X-Ray Pulses Directly in the Time Domain, Nat. Photonics 8, 950 (2014).

[43] G. De Ninno, D. Gauthier, B. Mahieu, P. Rebernik, E. Allaria, P. Cinquegrana, M. B. Danailov, A. Demidovich, E. Ferrari, L. Giannessi et al., Single-Shot SpectroTemporal Characterization of XUV Pulses from a Seeded Free-Electron Laser, Nat. Commun. 6, 8075 (2015).

[44] T. E. Glover, R. W. Schoenlein, A. H. Chin, and C. V. Shank, Observation of Laser Assisted Photoelectric Effect and Femtosecond High Order Harmonic Radiation, Phys. Rev. Lett. 76, 2468 (1996).

[45] J. M. Schins, P. Breger, P. Agostini, R. C. Constantinescu, H. G. Muller, A. Bouhal, G. Grillon, A. Antonetti, and A. Mysyrowicz, Cross-Correlation Measurements of Femtosecond Extreme-Ultraviolet High-Order Harmonics, J. Opt. Soc. Am. B 13, 197 (1996).

[46] A. Bouhal, R. Evans, G. Grillon, A. Mysyrowicz, P. Breger, P. Agostini, R. C. Constantinescu, H. G. Muller, and D. von der Linde, Cross-Correlation Measurement of Femtosecond Noncollinear High-Order Harmonics, J. Opt. Soc. Am. B 14, 950 (1997).

[47] E. Allaria, C. Callegari, D. Cocco, W. M. Fawley, M. Kiskinova, C. Masciovecchio, and F. Parmigiani, The FERMI@Elettra Free-Electron-Laser Source for Coherent X-Ray Physics: Photon Properties, Beam Transport System and Applications, New J. Phys. 12, 075002 (2010).

[48] L. H. Yu, Generation of Intense UV Radiation by Subharmonically Seeded Single-Pass Free-Electron Lasers, Phys. Rev. A 44, 5178 (1991).
[49] D. Gauthier, P. Rebernik, G. De Ninno, E. Allaria, P. Cinquegrana, M. B. Danailov, A. Demidovich, E. Ferrari, L. Giannessi, B. Mahieu, and G. Penco, Spectrotemporal Shaping of Seeded Free-Electron Laser Pulses, Phys. Rev. Lett. 115, 114801 (2015).

[50] B. Mahieu, D. Gauthier, G. De Ninno, H. Dacasa, M. Lozano, J.-P. Rousseau, P. Zeitoun, D. Garzella, and H. Merdji, Spectral-Phase Interferometry for Direct Electric-Field Reconstruction Applied to Seeded Extreme-Ultraviolet Free-Electron Lasers, Opt. Express 23, 17665 (2015).

[51] C. Iaconis and I. A. Walmsley, Spectral Phase Interferometry for Direct Electric-Field Reconstruction of Ultrashort Optical Pulses, Opt. Lett. 23, 792 (1998).

[52] A. A. Lutman, G. Penco, P. Craievich, and J. Wu, Impact of an Initial Energy Chirp and an Initial Energy Curvature on a Seeded Free Electron Laser: Free Electron Laser Properties, J. Phys. A 42, 085405 (2009).

[53] M. Labat, N. Joly, S. Bielawski, C. Szwaj, C. Bruni, and M. E. Couprie, Pulse Splitting in Short Wavelength Seeded Free Electron Lasers, Phys. Rev. Lett. 103, 264801 (2009).

[54] R. Bonifacio, L. De Salvo Souza, P. Pierini, and N. Piovella, The Superradiant Regime of a FEL: Analytical and Numerical Results, Nucl. Instrum. Methods Phys. Res., Sect. A 296, 358 (1990).

[55] R. Bonifacio, N. Piovella, and B. W. J. McNeil, Superradiant Evolution of Radiation Pulses in a Free-Electron Laser, Phys. Rev. A 44, R3441 (1991).

[56] L. Giannessi, P. Musumeci, and S. Spampinati, Nonlinear Pulse Evolution in Seeded Free-Electron Laser Amplifiers and in Free-Electron Laser Cascades, J. Appl. Phys. 98, 043110 (2005).

[57] T. Watanabe, X. J. Wang, J. B. Murphy, J. Rose, Y. Shen, T. Tsang, L. Giannessi, P. Musumeci, and S. Reiche, Experimental Characterization of Superradiance in a Single-Pass High-Gain Laser-Seeded Free-Electron Laser Amplifier, Phys. Rev. Lett. 98, 034802 (2007).

[58] The parameter $\rho_{\text {FEL }}$ is defined as $\rho_{\text {FEL }}=$ $\frac{1}{4 \pi \gamma}\left\{\left[\left(2 \pi^{2}\right) / \Sigma_{b}\right]\left(f_{b}(\xi) \lambda_{u} K\right)^{2}\left[\left(I_{\text {peak }}\right) / I_{A}\right]\right\}^{\frac{1}{3}}, \quad$ where $\quad K=$ $e_{0} \lambda_{u} B_{r m s} / 2 \pi c m_{0}$ is the strength of the undulator of the rms field $B_{r m s}$ ( $e_{0}$ being the electron charge, $c$ the speed of light, and $m_{0}$ the electron rest mass), $\Sigma_{b}$ is the electron beam cross section, $I_{\text {peak }}$ is the e-beam peak current, and $I_{A}=e_{0} c / r_{0} \sim 17 k A$ is the Alfven current (with $r_{0}$ the electron classical radius). The function $f_{b}(\xi)=$ $J_{0}(\xi)-J_{1}(\xi)$, where $\xi=K^{2} /\left(4+2 K^{2}\right)$, is a factor arising from the average over the longitudinal fast motion typical of the electron orbit in linear undulators. The parameter $\rho_{\text {FEL }}$ for a helical undulator is retrieved by setting $f_{b}(\xi)=\sqrt{2}$.

[59] R. Bonifacio, C. Pellegrini, and L. M. Narducci, Collective Instabilities and High-Gain Regime in a Free Electron Laser, Opt. Commun. 50, 373 (1984).

[60] P. Sprangle and A. T. Drobot, The Linear and SelfConsistent Nonlinear Theory of the Electron Cyclotron Maser Instability, IEEE Trans. Microwave Theory Tech. 25, 528 (1977).

[61] The coordinates $(z, \zeta)$ are the result of the transformation $z_{l}, t_{l} \rightarrow \zeta=z_{l}-\beta_{z} c t_{l}, \quad z=c t_{l}$, where $\left(z_{l}, t_{l}\right)$ represent 
space and time coordinates in the laboratory. Here, $z$ represents the position along the undulator and $\zeta$ a coordinate along the electron bunch moving at speed $\beta_{z} c$.

[62] G. Stupakov, Technical Report No. SLAC-PUB-14639, SLAC National Accelerator Laboratory, Stanford University, 2011.

[63] Equation (11) was derived in Ref. [64] by numerically fitting the first maximum of $J_{n}(x)$, and it is accurate to about $1 \%$ for $n \geqq 3$. A series expansion with arbitrary accuracy is given in Ref. [65], par. 9.5.16.

[64] L. Giannessi, Synchrotron Light Sources and FreeElectron Lasers Accelerators Physics, Instrumentation and Science Applications, edited by E. Jaeschke, S. Khan, J. R. Schneider, and J. B. Hastings (Springer International Publishing, Switzerland, 2015).

[65] M. Abramowitz and I. A. Stegun, Handbook of Mathematical Functions: With Formulas, Graphs, and Mathematical Tables (Dover Publications, New York, 1965).

[66] G. De Ninno, B. Mahieu, E. Allaria, L. Giannessi, and S. Spampinati, Chirped Seeded Free-Electron Lasers: SelfStanding Light Sources for Two-Color Pump-Probe Experiments, Phys. Rev. Lett. 110, 064801 (2013).

[67] B. Mahieu, E. Allaria, D. Castronovo, M. B. Danailov, G. Demidovich, A. De Ninno, S. Di Mitri, W. M. Fawley, E. Ferrari, L. Fröhlich et al., Two-Colour Generation in a Chirped Seeded Free-Electron Laser: A Close Look, Opt. Express 21, 22728 (2013).

[68] L. Giannessi, M. Artioli, M. Bellaveglia, F. Briquez, E. Chiadroni, A. Cianchi, M. E. Couprie, G. Dattoli, E. Di Palma, G. Di Pirro et al., High-Order-Harmonic Generation and Superradiance in a Seeded Free-Electron Laser, Phys. Rev. Lett. 108, 164801 (2012).

[69] D. Gauthier, B. Mahieu, and G. De Ninno, Direct Spectrotemporal Characterization of Femtosecond Extreme-Ultraviolet Pulses, Phys. Rev. A 88, 033849 (2013).

[70] K.-J. Kim and M. Xie, Self-Amplified Spontaneous Emission for Short Wavelength Coherent Radiation, Nucl. Instrum. Methods Phys. Res., Sect. A 331, 359 (1993).

[71] M. Xie, Design Optimization for an X-Ray Free Electron Laser Driven by SLAC Linac, in Proceedings of the 1995 Particle Accelerator Conference (Springer, 1995), Vol. 1, pp. 183-185.

[72] G. Dattoli, L. Giannessi, P. L. Ottaviani, and C. Ronsivalle, Semi-Analytical Model of Self-Amplified SpontaneousEmission Free-Electron Lasers, Including Diffraction and Pulse-Propagation Effects, J. Appl. Phys. 95, 3206 (2004).

[73] E. Allaria, L. Badano, S. Bassanese, F. Capotondi, D. Castronovo, P. Cinquegrana, M. B. Danailov, G. D'Auria, A. Demidovich, R. De Monte et al., The FERMI FreeElectron Lasers, J. Synchrotron Radiat. 22, 485 (2015).

[74] M. Zangrando, A. Abrami, D. Bacescu, I. Cudin, C. Fava, F. Frassetto, A. Galimberti, R. Godnig, D. Giuressi, L. Poletto et al., The Photon Analysis, Delivery, and Reduction System at the FERMI@Elettra Free Electron Laser User Facility, Rev. Sci. Instrum. 80, 113110 (2009).

[75] M. Zangrando, D. Cocco, C. Fava, S. Gerusina, R. Gobessi, N. Mahne, E. Mazzucco, L. Raimondi, L. Rumiz, and C. Svetina, Recent Results of Padres, the Photon Analysis Delivery and Reduction System, from the FERMI
FEL Commissioning and User Operations, J. Synchrotron Radiat. 22, 565 (2015).

[76] L. Raimondi, C. Svetina, N. Mahne, D. Cocco, A. Abrami, M. De Marco, C. Fava, S. Gerusina, R. Gobessi, F. Capotondi et al., Microfocusing of the FERMI@Elettra FEL Beam with a K-B Active Optics System: Spot Size Predictions by Application of the WISE Code, Nucl. Instrum. Methods Phys. Res., Sect. A 710, 131 (2013).

[77] P. Kirkpatrick and A. V. Baez, Formation of Optical Images by X-Rays, J. Opt. Soc. Am. 38, 766 (1948).

[78] C. Svetina, C. Grazioli, N. Mahne, L. Raimondi, C. Fava, M. Zangrando, S. Gerusina, M. Alagia, L. Avaldi, G. Cautero et al., The Low Density Matter (LDM) Beamline at FERMI: Optical Layout and First Commissioning, J. Synchrotron Radiat. 22, 538 (2015).

[79] F. Capotondi, E. Pedersoli, N. Mahne, R. H. Menk, G. Passos, L. Raimondi, C. Svetina, G. Sandrin, M. Zangrando, M. Kiskinova et al., Invited Article: Coherent Imaging Using Seeded Free-Electron Laser Pulses with Variable Polarization: First Results and Research Opportunities, Rev. Sci. Instrum. 84, 051301 (2013).

[80] F. Capotondi, E. Pedersoli, F. Bencivenga, M. Manfredda, N. Mahne, L. Raimondi, C. Svetina, M. Zangrando, A. Demidovich, I. Nikolov et al., Multipurpose End-Station for Coherent Diffraction Imaging and Scattering at FERMI@Elettra Free-Electron Laser Facility, J. Synchrotron Radiat. 22, 544 (2015).

[81] M. B. Danailov, F. Bencivenga, F. Capotondi, F. Casolari, P. Cinquegrana, A. Demidovich, E. Giangrisostomi, M. P. Kiskinova, G. Kurdi, M. Manfredda et al., Towards JitterFree Pump-Probe Measurements at Seeded Free Electron Laser Facilities, Opt. Express 22, 12869 (2014).

[82] P. Cinquegrana, S. Cleva, A. Demidovich, G. Gaio, R. Ivanov, G. Kurdi, I. Nikolov, P. Sigalotti, and M. B. Danailov, Optical Beam Transport to a Remote Location for Low Jitter Pump-Probe Experiments with a Free Electron Laser, Phys. Rev. ST Accel. Beams 17, 040702 (2014).

[83] V. Véniard, R. Taïeb, and A. Maquet, Two-Color Multiphoton Ionization of Atoms Using High-Order Harmonic Radiation, Phys. Rev. Lett. 74, 4161 (1995).

[84] M. Meyer, J. T. Costello, S. Düsterer, W. B. Li, and P. Radcliffe, Two-Colour Experiments in the Gas Phase, J. Phys. B 43, 194006 (2010).

[85] W. Li, X. Ma, X. Yang, and Z. Wang, Simulation of CrossCorrelation Method for Temporal Characterization of VUV Free-Electron-Lasers, Chin. Optic. Lett. 11, 091403 (2013).

[86] We use $\tau_{\mathrm{FEL}}$ to indicate the "full with half maximum" (FWHM) duration and $\sigma^{\mathrm{FEL}}$ to indicate the rms duration, defined in Eq. (12). The extraction of the rms value from experimental data is prone to systematic errors due to the effects of the background noise. For this reason, experimental data are provided as FWHM. For a Gaussian distribution, $\tau_{\mathrm{FEL}} \simeq 2.35 \sigma^{\mathrm{FEL}}$.

[87] A. Maquet and R. TaIeb, Two-Colour IR + XUV Spectroscopies: The "Soft-Photon Approximation", J. Mod. Opt. 54, 1847 (2007).

[88] M. Mucke, V. Zhaunerchyk, L. J. Frasinski, R. J. Squibb, M. Siano, J. H. D. Eland, P. Linusson, P. Salén, P. van der 
Meulen, R. D. Thomas et al., Covariance Mapping of Two-Photon Double Core Hole States in $\mathrm{C}_{2} \mathrm{H}_{2}$ and $\mathrm{C}_{2} \mathrm{H}_{6}$ Produced by an X-Ray Free Electron Laser, New J. Phys. 17, 073002 (2015).

[89] C. Svetina, A. Abrami, I. Cudin, C. Fava, S. Gerusina, R. Gobessi, L. Rumiz, G. Sostero, M. Zangrando, and D. Cocco, Characterization of the FERMI@Elettra On-Line Photon Energy Spectrometer, Proc. SPIE Int. Soc. Opt. Eng. 8139, 81390J (2011).

[90] U. Teubner, U. Wagner, and E. Förster, Sub-10 fs Gating of Optical Pulses, J. Phys. B 34, 2993 (2001).

[91] C. Gahl, A. Azima, M. Beye, M. Deppe, K. Döbrich, U. Hasslinger, F. Hennies, A. Melnikov, M. Nagasono, A. Pietzsch et al., A Femtosecond X-Ray/Optical CrossCorrelator, Nat. Photonics 2, 165 (2008).

[92] M. Beye, O. Krupin, G. Hays, A. H. Reid, D. Rupp, S. de Jong, S. Lee, W.-S. Lee, Y.-D. Chuang, R. Coffee et al., X-Ray Pulse Preserving Single-Shot Optical CrossCorrelation Method for Improved Experimental Temporal Resolution, Appl. Phys. Lett. 100, 121108 (2012).

[93] O. Krupin, M. Trigo, W. F. Schlotter, M. Beye, F. Sorgenfrei, J. J. Turner, D. A. Reis, N. Gerken, S. Lee, W. S. Lee et al., Temporal Cross-Correlation of X-Ray Free Electron and Optical Lasers Using Soft X-Ray Pulse Induced Transient Reflectivity, Opt. Express 20, 11396 (2012).

[94] M. Harmand, R. Coffee, M. R. Bionta, M. Chollet, D. French, D. Zhu, D. M. Fritz, H. T. Lemke, N. Medvedev, B. Ziaja et al., Achieving Few-Femtosecond Time-Sorting at Hard X-Ray Free-Electron Lasers, Nat. Photonics 7, 215 (2013).

[95] F. Casolari, F. Bencivenga, F. Capotondi, E. Giangrisostomi, M. Manfredda, R. Mincigrucci, E. Pedersoli, E. Principi, C. Masciovecchio, and M. Kiskinova, Role of Multilayer-Like Interference Effects on the Transient Optical Response of $\mathrm{SI}_{3} \mathrm{~N}_{4}$ Films Pumped with Free-Electron Laser Pulses, Appl. Phys. Lett. 104, 191104 (2014).

[96] T. Maltezopoulos, S. Cunovic, M. Wieland, M. Beye, A. Azima, H. Redlin, M. Krikunova, R. Kalms, U. Frühling, F. Budzyn et al., Single-Shot Timing Measurement of Extreme-Ultraviolet Free-Electron Laser Pulses, New J. Phys. 10, 033026 (2008).

[97] S. Schorb, T. Gorkhover, J. P. Cryan, J. M. Glownia, M. R. Bionta, R. N. Coffee, B. Erk, R. Boll, C. Schmidt, D. Rolles et al., X-Ray-Optical Cross-Correlator for GasPhase Experiments at the Linac Coherent Light Source Free-Electron Laser, Appl. Phys. Lett. 100, 121107 (2012).

[98] N. Medvedev, B. Ziaja, M. Cammarata, M. Harmand, and S. Toleikis, Electron Kinetics in Femtosecond X-Ray Irradiated $\mathrm{SiO}_{2}$, Contrib. Plasma Phys. 53, 347 (2013).

[99] B. Rethfeld, A. Rämer, N. Brouwer, N. Medvedev, and O. Osmani, Electron Dynamics and Energy Dissipation in
Highly Excited Dielectrics, Nucl. Instrum. Methods Phys. Res., Sect. B 327, 78 (2014).

[100] S. J. Orfanidis, Electromagnetic Waves and Antennas (Rutgers University Press, New Brunswick, 2013).

[101] L. Spitzer, Jr., Physics of Fully Ionized Gases (Wiley Interscience, New York, 1962).

[102] I. P. Shkarofsky, T. W. Johnston, and M. P. Bachynski, The Particle Kinetics of Plasmas (Addison-Wesley, Boston, 1966).

[103] N. Medvedev and B. Rethfeld, Effective Energy Gap of Semiconductors under Irradiation with an Ultrashort VUV Laser Pulse, Europhys. Lett. 88, 55001 (2009).

[104] N. Medvedev, Femtosecond X-Ray Induced Electron Kinetics in Dielectrics: Application for FEL-PulseDuration Monitor, Appl. Phys. B 118, 417 (2015).

[105] E. Riedle, M. Beutter, S. Lochbrunner, J. Piel, S. Schenkl, S. Spörlein, and W. Zinth, Generation of 10 to $50 \mathrm{fs}$ Pulses Tunable through All of the Visible and the NIR, Appl. Phys. B 71, 457 (2000).

[106] D. Grojo, M. Gertsvolf, S. Lei, T. Barillot, D. M. Rayner, and P. B. Corkum, Exciton-Seeded Multiphoton Ionization in Bulk $\mathrm{SiO}_{2}$, Phys. Rev. B 81, 212301 (2010).

[107] P. Martin, S. Guizard, Ph. Daguzan, G. Petite, P. D'Oliveira, P. Meynadier, and M. Perdrix, Subpicosecond Study of Carrier Trapping Dynamics in Wide-Band-Gap Crystals, Phys. Rev. B 55, 5799 (1997).

[108] S. Eckert, M. Beye, A. Pietzsch, W. Quevedo, M. Hantschmann, M. Ochmann, M. Ross, M. P. Minitti, J. J. Turner, S. P. Moeller et al., Principles of Femtosecond $X$-Ray/Optical Cross-Correlation with X-Ray Induced Transient Optical Reflectivity in Solids, Appl. Phys. Lett. 106, 061104 (2015).

[109] S. K. Sundaram and E. Mazur, Inducing and Probing Non-thermal Transitions in Semiconductors Using Femtosecond Laser Pulses, Nat. Mater. 1, 217 (2002).

[110] B. L. Henke, E. M. Gullikson, and J. C. Davis, X-Ray Interactions: Photoabsorption, Scattering, Transmission, and Reflection at $E=50-30,000 \mathrm{ev}, Z=1-92$, At. Data Nucl. Data Tables 54, 181 (1993).

[111] M. Drescher, U. Frühling, M. Krikunova, T. Maltezopoulos, and M. Wieland, Time-Diagnostics for Improved Dynamics Experiments at XUV FELs, J. Phys. B 43, 194010 (2010).

[112] J. Boedewadt and C. Lechner, Results and Perspectives on the FEL Seeding Activities at Flash, in Proceedings of FEL2013, New York, NY, USA (JACoW, 2013), p. WEPSO02.

[113] T. Zhang, D. Wang, Z. Zhao, and X. Yang, Design of a Wavelength Continuously Tunable Ultraviolet Coherent Light Source, in Proceedings of IPAC2012, New Orleans, Louisiana, USA (IEEE, 2012), p. TUPPP057. 